\title{
Research Article \\ Dynamic Retransmission Limit Scheme in MAC Layer for Routing in Multihop Ad hoc Networks
}

\author{
Ralph El-Khoury and Rachid El-Azouzi \\ LIA/CERI, University of Avignon, Agroparc, BP 1228, 84911 Avignon Cedex 9, France \\ Correspondence should be addressed to Rachid El-Azouzi, rachid.elazouzi@univ-avignon.fr
}

Received 27 January 2008; Accepted 20 April 2008

Recommended by Tarik Taleb

\begin{abstract}
We consider a wireless ad hoc network with random access channel. We present a model that takes into account topology, routing, random access in MAC layer, and forwarding probability. In this paper, we focus on drawing benefit from the interaction of the MAC (governed by IEEE 802.11 or slotted Aloha) and routing by defining a new cross-layer scheme for routing based on the limit number of retransmission. By adjusting dynamically and judiciously this parameter in a saturated network, we have realized that both stability of forwarding queues and average throughput are significantly improved in linear networks with symmetric traffic: a gain of $100 \%$ can be reached. While in asymmetric topology network with asymmetric traffic, we achieve a better average delay (resp., throughput) for each connection without changing the average throughput (resp., delay). In addition, we show the efficiency of our new scheme in case of multimedia applications with delay constraint. A detailed performance study is presented using analytical and simulation evaluation.
\end{abstract}

Copyright (C) 2008 R. El-Khoury and R. El-Azouzi. This is an open access article distributed under the Creative Commons Attribution License, which permits unrestricted use, distribution, and reproduction in any medium, provided the original work is properly cited.

\section{INTRODUCTION}

A multihop wireless ad hoc network is a collection of nodes that communicate with each other without any established infrastructure or centralized control. Each of these nodes is a wireless transceiver that transmits and receives at a singlefrequency band which is common to all the nodes. These nodes can communicate with each other, however, they are limited by their transmitting and receiving capabilities. Therefore, they cannot directly reach all of the nodes in the network as most of the nodes are outside of direct range. In such a scenario, one of the possibilities for the information transmission between two nodes that are not in position to have a direct communication is to use other nodes in the network. To be precise, the source node transmits its information to one of the nodes which is within transmission range of the source node. In order to overcome this, the network operates in a multihop fashion. Nodes route traffic for each other. Therefore, in a connected ad hoc network, a packet can travel from any source to its destination either directly, or through some set of intermediate packet forwarding nodes.
In recent years, an increased effort was consecrated to cross-layer design, of ad hoc networks, where information is exchanged between different layers. In wireless context where channel conditions and network connectivity impose serious challenges, new cross-layer approaches are needed to optimize performances. In fact, the knowledge of channel condition in the physical layer may help on choosing the adequate number of retransmission in the MAC layer which also depends on the application requirement and the transport layer utilized. On the one hand, it is also profitable for routing protocols to know the link state of lower layers to choose the best route possible in terms of available bandwidth, stability of links, energy consumptions, and so on. On the other hand, giving route information to the MAC layer can be efficient to control packet retransmissions, as we will see in this paper. In this case, we can achieve better QoS in terms of end-toend delay and throughput. Moreover, by using cross-layer approaches, we can avoid network partitioning due to both link degradation (and thus link breakage) and rapid energy expiration. Various cross-layering approaches are analyzed in [1-3]. 
To study the network performances with the interaction of various parameters from different layers, we consider in this paper the framework of random access mechanism for the wireless channel where the nodes having packets to transmit in their buffers attempt transmissions by delaying the transmission by a random amount of time. This mechanism acts as a way to avoid collisions of transmissions of nearby nodes in the case where nodes cannot sense the channel while transmitting (hence, are not aware of other ongoing transmissions). We assume that time is slotted into fixed length time frames. In any slot, a node having a packet to be transmitted to one of its neighboring nodes decides with some fixed (possibly node-dependent) probability in favor of a transmission attempt. If there is no other transmission by the other nodes whose transmission can interfere with the node under consideration, the transmission is successful. We assume throughout that there is some mechanism that notifies the sender of success or failure of its transmissions. For example, the sources get the feedback on whether there was zero, one, or more transmissions (collision) during the time slot.

At any instant in time, a node may have two kinds of packets to be transmitted as follows.

(1) Packets generated by the node itself. This can be sensed data if we consider a sensor network.

(2) Packets from other neighboring nodes that need to be forwarded.

In this paper, we consider two separate queues for these two types and do a weighted fair queueing (WFQ) for these two queues. This type of configuration allows us to include in the model the cooperation level which represents the fraction of the traffic forwarded by a node in ad hoc network.

In $[4,5]$, working with the above-mentioned system model, we have already studied the impact of routing, channel access rates and weights of the weighted fair queueing on throughput, stability, and fairness properties of the network. We obtained important insights into various tradeoffs that can be achieved by varying certain network parameters. The throughput maximization of the multihop wireless networks has been extensively studied in [6-8]. However, it is shown that the high throughput in the ad hoc network is achieved at the cost of a high amount of delay. This problem has drawn our attention to the relation between the delay characteristic and the throughput.

In this paper, we use a cross layer optimization between MAC and network layer for routing. For a given path between a source and a destination, each intermediate node computes a new limit number of retransmission based on a specific algorithm. This parameter can be adjusted easily by each node. Using this new routing, we achieve a better average delay (resp., throughput) for each connection without changing the average throughput (resp., delay). In extreme cases, a reset technique is introduced to optimize performances. In addition, we show the efficiency of our scheme in case of multimedia applications with delay constraint.

\section{Related work}

In most recent literature, the tradeoffs between throughput and delay have been investigate as a key measure of the network performance. Several studies have first focused on wireless network stability and finding the maximum achievable throughput. Stability problem was considerably studied for the Aloha $[11,12]$ and scheduling $[9,10]$ protocols. Tassiulas and Ephremides [9] obtain a scheduling policy for the nodes that maximizes the stability region. Their approach inherently avoids collisions which allows to maximize the throughput. Radunovic and Le Boudec [13] suggest that considering the total throughput as a performance objective may not be a good objective. Moreover, most of the related studied did not consider the problem of forwarding and each flow is treated similarly (except for Radunovic and Le Boudec [13], Huang and Bensaou [14], or Tassiulas and Sarkar [15]). Our setting is different from the mentioned ones in the following: the number of retransmission is finite, and therefore in our setting, the output and the input rates need not be the same.

In recent years, there has been a considerable effort on trying to increase the performance of wireless ad hoc networks since Gupta and Kumar [7] showed that the capacity of a fixed wireless network decreases as the number of nodes increases. Grossglauser and Tse [6] presented a two-phase packet forwarding technique for mobile ad hoc networks, utilizing the multiuser diversity, in which a source node transmits a packet to the destination when this destination becomes the closet neighbors of the relay. This scheme was shown to increase the capacity of the MANET, such that it remains constant as the number of users in the MANET increases. However, the delay experienced by packet under this strategy was shown to be large and it can be even infinite for a fixed number of nodes in the system. Since both capacity as well as delay are important from an application point of view, many works have recently been studied to understand the delay-capacity relationship in ad hoc networks. In [1], the authors considered wireless network consisting of static sender-destination pairs and mobile relays, and proposed a geographic routing scheme that achieves near optimal capacity. They showed that under this scheme, the near optimal capacity is achieved with the low delay. Many papers have studied the tradeoff between throughput and delay. In $[16,17]$, the authors achieve a high throughput and low delay in adhoc networks. El Gamal et al. [18] analyze the optimal delay-throughput scaling for different wireless network topologies. In the static random network with $n$ nodes, they obtain an optimal tradeoff between throughput and delay. Neely et al. [19] consider the delay-throughput tradeoff only for mobile ad hoc networks.

The problem of optimizing the tradeoff throughput delay can have a direct impact on the QoS of multimedia applications. The previous mentioned literatures have mainly worked on analytical models. Moreover, many papers have proposed some heuristics and new methods to improve the quality of real-time streaming media over wireless networks. Since the wireless links cause a lot of challenges due to 
the variation of channel conditions, authors in [20-22] have treated this problem by proposing some algorithms to adapt video streaming to the wireless channel in a WLAN. Their ideas are based on protecting high-priority packets and on considering the variation of channel conditions. Authors in [20] propose a selective packet algorithm that runs in the base-station to determine which of the delayed packets to deliver. Packets that have missed their play-out time are removed from the base-station buffer without being transmitted. In [21], authors developed a cross-layer algorithm motivated by the advantage of adapting the retry limit. They observed that this latter can be optimized in such a way that the overall packet losses from link errors and buffer overflow are minimized. In addition, high priority packets are protected by giving them higher retry limit. In [22], a retransmission deadline is assigned to each packet by the application layer. Therefore, the MAC layer can decide when to drop the packet by adapting the retry limit parameter in the IEEE 802.11. It is important in such mechanism to find the good optimization for the existing tradeoff between the delay incurred by increasing the retry limit to protect some packets and the packet loss due to a late arrival.

In multihop networks case, the QoS problem is more complicated as it includes additional factors like multihop transmissions, variation of the channel conditions, link breakage, congestion. Papers in [23-26] have studied the problem of real-time video over ad hoc networks. Authors in [23] believed that multipath transport is a promising technique for this problem. In [24], a cross-layer approach (including APP/NETWORK/MAC layers) based on adaptive rate control for video streaming is proposed. Source nodes must estimate the minimum effective transmission rate of a given path to decide on their rate. The endto-end QoS was improved, but the challenge is to find the good balance between performance gains and design complexity as mentioned in [25]. In [26], it is shown how the multihop paths and link failure can influence the performances, and therein authors suggest a mechanism to adapt the application layer to network topology change.

\section{Contribution}

In comparison to previous works, the following distinguishes our paper.

(i) We use a multilayer model that can track the retransmission effects on the performances. Then, in symmetric networks, it is possible to prove the efficiency of any methods that control the retransmission limit.

(ii) Encouraged by previous studies done in WLAN on the retransmission limit, we remarked that an optimization of this latter through a given path in multihop networks can increase the available bandwidth. It is a kind of congestion control that can be applied to multimedia streams. (iii) The distributed cross-layer scheme that we propose besides of its novelty and efficiency is characterized by its simplicity. It does not need external information to the node itself, but a local decision can be taken with the help of routing information from the network layer and load estimation if needed.

The rest of the paper is organized as follows. In Section 2, we describe the network model. Then in Section 3, we present our new cross-layer dynamic scheme for routing. A detailed evaluation of performance is performed in Section 4 to show the efficiency of our new scheme. In Section 5, we study the performances in the case of delay-sensitive connections. Finally, we give a conclusion in Section 6.

\section{NETWORK MODEL}

\subsection{Assumptions and definitions}

We model the ad hoc wireless network as a set of $N$ nodes deployed arbitrarily in a given area. We assume the following.

(i) A single channel. Nodes use the same frequency for transmitting with an omnidirectional antenna. A node $j$ receives successfully a packet from a node $i$ if and only if there is no interference at the node $j$ due to another transmission on the same channel, that is, if there is no transmission from any node of the set $N(j) \cup j$, where $N(j)$ is the set of neighbors of node $j$. We assume that all the nodes in $N(j)$ has $j$ as a neighbor. Note also that a node cannot receive and transmit at the same time.

(ii) Two types of queues. Two queues are associated with each node. The first one is the forwarded queue, noted by $F_{i}$ (proper to the node $i$ ), which carries all the packets originated from a given source and destined to a given destination. The second is $Q_{i}$ which carries the proper packets of the node $i$ (in this case $i \equiv s$, where $s$ designates a source node). We assume that each node has an infinite capacity of storage for the two queues. When $F_{i}$ has a packet to be sent, the node chooses to send it from $F_{i}$ with a probability $f_{i}$. In other terms, it chooses to send from $Q_{i}$ with probability $1-f_{i}$. When one of these queues is empty, then we choose to send a packet from the nonempty one with a probability 1 .

(iii) Saturated network. Each node has always packets to be sent from queue $Q_{i}$, whereas $F_{i}$ can be empty. Consequently, the network is considered saturated, and thus it depends on the channel access mechanism. This assumption is suitable to determine the limit operation of the network. Besides, analytical results become feasible.

\subsection{Network layer}

Network layer handles the two queues $Q_{i}$ and $F_{i}$ using the WFQ scheme, as described previously. Also, this layer maintains routing algorithms. So, each node acts as a router, 
it permits to relay packets originated from a source $s$ to a destination $d$. It must carry a routing information which permits sending of packets to a destination via a neighbor. In this paper, we assume that nodes form a static network where routes between any source $s$ and destination $d$ are invariant in the saturated network case. Proactive routing protocols as optimized link state routing (OLSR) construct and maintain a routing table that carries routes to all nodes on the network. This kind of protocols corresponds well with our model. Note that the set of nodes between a node $s$ and $d$ is designated by $R_{s, d}$.

\subsection{MAC layer}

We assume a channel access mechanism only based on a probability to access the network, that is, when a node $i$ has a packet to transmit from the queue $Q_{i}$ or $F_{i}$, it accesses the channel with a probability $P_{i}$. For example, in IEEE 802.11 distributed coordination function (DCF), the attempt probability is given by [27]

$$
P=\frac{2\left(1-2 P_{c}\right)}{\left(1-2 P_{c}\right)\left(C W_{\min }+1\right)+P_{c} C W_{\min }\left(1-\left(2 P_{c}\right)^{m}\right)},
$$

where $P_{c}$ is the conditional collision probability given that a transmission attempt is made, and $m=\log _{2}\left(C W_{\max } /\right.$ $\left.C W_{\min }\right)$ is the maximum of back-off stage. $C W_{\max }$ and $C W_{\text {min }}$ designate the maximum and the minimum contention windows, respectively. The scheduler of transmission overall the network depends on $P_{i}$. We assume that each node is notified about the success or failure of its transmitted packets. A packet is failure only when there is an interference on the intended receiver, in other terms, when a collision occurs on the receiver. We have considered previously infinite buffer size, therefore, there is no packet loss due to overflow at the queues. The only source of packet loss is due to collisions. For a reliable communication, we allow a limit number of successive transmissions for a packet, after that it will be dropped definitively. Considering infinite forwarding buffer (yet neglecting the losses due to overflow) does not really affect our results because we are considering stability issue. In fact, this latter gives a practical indication on the congestion occurring on the network.

\subsection{Layer representation of the model}

The model of Figure 1 is a layer representation of our model for each node. Attempting to access the channel begins by choosing the queue from which a packet must be selected. And then, this packet is moved from the corresponding queue from the network layer to the MAC layer where it will be transmitted and retransmitted, if needed, until its success or drop. In this manner, when a packet is in the MAC layer, it is itself attempted successively until it is removed from the node.

\subsection{Main notations}

We summarize the parameters and notations used in this paper for a general network topology.

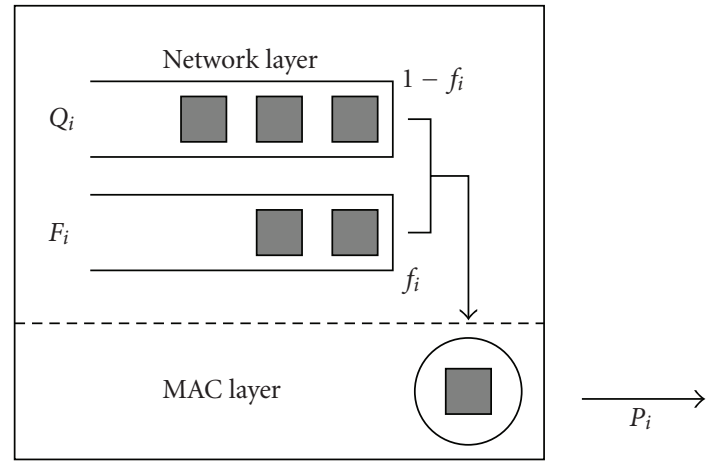

FIgURE 1: Network layer and MAC layer of node $i$.

(1) MAC layer notations are as follows.

(a) $P_{i}$ is the probability of transmission on the channel of the node $i$.

(b) $P_{i, s, d}$ is the probability that a transmission from node $i$ on the path from $s$ to $d$ is successful.

(c) $K_{i, s, d}$ is the maximum number of transmissions allowed to a packet sent from the node $i$ on the path from $s$ to $d$. After $K_{i, s, d}$ failures, the packet is dropped.

(d) $L_{i, s, d}$ is the expected number of attempts till successful or a drop from node $i$ on the path from $s$ to $d$.

(2) Network layer notations are as follows.

(a) $f_{i}$ is the probability to send a packet from the queue $F_{i}$ when it carries a packet.

(b) $R_{s, d}$ is the set of intermediate nodes in a path between a node $s$ and a node $d . s$ and $d$ are not in this set.

(c) $R_{i, s, d}$ is the set of nodes $R_{s, i} \cup i$ in the path $s, d$.

(d) $j_{i, s, d}$ designates a neighbor node of $i$ that comes after $i$ in the set $R_{s, d}$ toward the destination on the path from $s$ to $d$. It is the next hop node of the node $i$.

(e) $\pi_{i}$ is the probability that the queue $F_{i}$ has at least one packet to be forwarded after a departure of a packet. It is the load of the forwarding queue $F_{i} . y_{i}:=1-\pi_{i} f_{i}$ gives an indication about load or stability of queue $F_{i}$. Interesting results about the stability of forwarding queues were presented in our previous works $[4,5]$.

(f) $\pi_{i, s, d}$ is the probability that the queue $F_{i}$ has a packet at the first position ready to be forwarded to the path $R_{s, d}$ after a departure of a packet. It represents the intensity of traffic for a given path $R_{s, d}$.

(g) $P_{i, d}$ is the probability that the node $i$ chooses the path $R_{i, d}$ (whose destination is $d$ ) for sending packets from $Q_{i}$. Normally, this parameter can be assigned to the node $i$-transport layer decision.

\section{A NEW DYNAMIC SCHEME FOR THE MAXIMUM NUMBER OF TRANSMISSIONS IN ROUTING}

The maximum number of the transmissions $K_{i, s, d}$ of each node $i$ on a path $R_{s, d}$ appears to be an important parameter 
(in $[4,5]$ ) that can be adjusted easily by each node. When all nodes have the same static value $K$, it is sufficient to increase $K$ so that throughput is considerably ameliorated but the load is not. A tradeoff stability throughput is clearly noticed. Is it possible to benefit from a dynamic value of $K_{i, s, d}$ to optimize this tradeoff?

In this section, we propose a new dynamic $K_{i, s, d}$ scheme based on a table-driven routing where routes are already known. It is a cross-layer scheme where each node needs the information about the route to determine the corresponding $K_{i, s, d}$ which is a MAC layer parameter. Each node in a path $R_{s, d}$ must be introduced with the following:

(1) the length of the path in number of hops; and

(2) its position in the path in terms of the number of hops that separates it from the source.

These two pieces of information can be provided from the routing protocol. If they are available, our scheme computes the corresponding $K_{i, s, d}$. The following is a description of the scheme.

Consider that each node has a default value of the maximum number of transmissions set to $K$. Each node $i$ in the set $R_{s, d} \cup\{s\}$ computes the corresponding $K_{i, s, d}$ in such a manner that this latter is higher than or equal to the previous $K_{j, s, d}$, where $j$ is the previous node of $i$ in the path $R_{s, d} \cup\{s\}$, that is, $i=j_{j, s, d}$. Furthermore, the average values of $K_{i, s, d}$ (for $i \in R_{s, d} \cup\{s\}$ ) must be set to $K$, that is, $\left(1 /\left|R_{s, d} \cup\{s\}\right|\right) \sum_{i \in R_{s, d} \cup\{s\}} K_{i, s, d}=K$. Also, the values of $K_{i, s, d}$ (for $i \in R_{s, d} \cup\{s\}$ ) are determined based on the position of the node $i$ in the path $R_{s, d}$, that is, it is based on the number of hops that separates it from the source or the destination. We add to this scheme a reset technique when the average queue size or the load of $F_{i}$ exceeds some threshold. In fact, when the average queue value in dynamic case becomes not profitable in comparison to the static case, we reset the value of $K_{i, s, d}$ to $K$, or to a lower value. In the following section, we will specify a detailed and practical method for choosing $K_{i, s, d}$.

Under this scheme, we aim to give more chance of success to packets that had come near to their destination. It rather means that we need to avoid as much as possible loosing packets near their destination so that waste of bandwidth throughout a path becomes lower. In other terms, we expect to reduce the number of wasted slots in each connection.

Normally, the way of choosing good $K_{i, s, d}$ should depend on many parameters, not only on the number of hops of each connection but also on the transmission probabilities and the number of neighbors. Taking aware of many parameters at the same time is a complex issue. In this paper, we focus mainly on varying $K_{i, s, d}$ function of the number of hops. The following evaluation of performance section may clarify the interest of using dynamic $K_{i, s, d}$.

\section{PERFORMANCE EVALUATION: NUMERICAL RESULTS AND SIMULATIONS}

In this section, we evaluate the performance of the dynamic scheme for a symmetric linear and an asymmetric network.
We specify and detail our dynamic scheme for choosing $K_{i, s, d}$ in terms of the hop number. Then an analysis of the numerical and simulation results are given. All numerical results have been validated with our discrete time simulator that is presented in paper [5].

\subsection{Symmetric linear network case}

\subsubsection{Description of the network}

Our purpose by studying the linear network case is to understand the advantage of the new dynamic scheme so that we can understand the efficiency of the method on the asymmetric case. Also, the linear symmetric network is simple to study [4]. The characteristics of this network are as follows: (1) equal number of neighbors, that is, $n_{i}=2$ for all nodes; (2) $P_{i}=P$; (3) infinite number of nodes; (4) equal forwarding probability, that is, $f_{i}=f ;(5) P_{s, d}$, depends on the connection hop number of $h$, thus $P_{s, d}=P(h) ;(6)$ there is only one path between two different nodes, that is, one way to reach any destination from any source. The consequence of these hypothesis is that the stability is the same for all nodes, that is, $y_{i}=y$. When we take a uniform probability to choose a given path of length $h$, then $P(h)=1 / 2 B$, where $B$ represents the maximum number of hops to reach the farthest destination.

\subsubsection{Main expressions for performance evaluation}

We will mainly study the stability-throughput issue with the new scheme. In addition, the probability of success and the average number of transmissions bring additional material to understand what is happening in the network. Later, in the asymmetric network section, the end-to-end delay gives more information about stability of connections; while in the symmetric linear network, the stability of a node is equivalent for all nodes, thus the variation of a node stability informs about delay variation.

General expressions for performance evaluation in general networks were already derived in our paper [5], but in the linear symmetric case, these expressions can be derived from [4]. Here, we include the fact that the limit number of transmissions is the function of the number of hops. Let $T:=1-P_{i, s, d}$ be the collision probability, $P_{i, s, d}=(1-P)^{2}$, and $K(i, h)$ represents the value of $K_{i, s, d}$ in $K(i, h)$ notation, $i$ represents the position of the node on the path $R_{s, d}$. For example, $K_{s, s, d}=K(1, h), K_{j_{s, s, d}, s, d}=K(2, h)$, and so on. Let $L(i, h)$ be the average number of transmissions (i.e., $L_{i, s, d}$ ) of the node $i$ on the path of length $h$. Thus, $L(i, h)=$ $\left(1-T^{K(i, h)}\right) /(1-T)$. For all paths, the average number of transmissions $L(i, h)$ becomes

$$
L=2 \frac{(1-y)}{B^{2}-B} \sum_{j=1}^{B} \sum_{h=j+1}^{B} L(j, h)+2 y \sum_{h=1}^{B} P(h) L(1, h),
$$

where $\left(B^{2}-B\right)$ is the number of connections that traverse a node. Note that $L$ depends only on $y$ and $B$ for a given $P$. It means that $L$ for the dynamic case is different from the static one. 
Now, to determine $y$, we can write the balance rate equation of the forwarding queue. Then, the arrival rate to the forwarding queue of a given node is equal to the departure rate from it. The arrival rate from all sources of the network to a given node can be written as

$$
a=2 y \sum_{j=1}^{B} \sum_{h=j+1}^{B} P(h) \frac{P}{L} \prod_{k=1}^{j}\left(1-T^{K(k, h)}\right) .
$$

This latter is formed by two main parts: the first one is the traffic intensity of source nodes (of new packets) and the second one is the end-to-end probability of success. The departure rate is

$$
d=(1-y) \frac{P}{L}
$$

This latter also is formed by two parts: the first one is the intensity of forwarding packets $(1-y)$ and the second is the rate of removing a packet from the node by successful transmission or a drop after $K$ transmissions. Therefore, the stability region can be written as

$$
y=\frac{1}{1+2 \sum_{j=1}^{B} \sum_{h=j+1}^{B} P(h) \prod_{k=1}^{j}\left(1-T^{K(k, h)}\right)} .
$$

The throughput on the path of length $h$ can be written as

$$
\operatorname{thp}(h)=y P(h) \frac{P}{L} \prod_{k=1}^{h}\left(1-T^{K(k, h)}\right) .
$$

The average throughput for all $h$ is

$$
\overline{\operatorname{thp}}=y \frac{P}{B \cdot L} \cdot \sum_{h=1}^{B} P(h) \prod_{k=1}^{h}\left(1-T^{K(k, h)}\right) .
$$

The probability of success between a couple of nodes on a path of length $h$ is $\operatorname{Psucc}(h)=\prod_{k=1}^{h}\left(1-T^{K(k, h)}\right)$. The average probability of success for all $h$ is $\overline{P s u c c}=$ $(1 / B) \sum_{h=1}^{B} P \operatorname{succ}(h)$.

\subsubsection{A practical description of a dynamic method}

We use a simple method that gives a dynamic value of $K(i, h)$ depending on the length of a connection $h$, on the position of the node $i$ on the path $R_{s, d}$, and on $K^{\prime}$ the step of how much we increase $K$ on a given node $i$. Each node $i$ initializes $K(i, h)$ to $K$.

This method maintains an average $K$ of all $K(i, h)$ (for $\left.i \in R_{s, d} \cup\{s\}\right)$ values of each connection. For example, this average $K$ can be the default value of the maximum number of transmissions in a network of static $K$. Also, in this manner it will be easy to compare the static $K$ case performance and the dynamic one.

Practically, for each packet transmission, a node $i$ must know its $K(i, h)$ on a given path (or connection) of length $h$. In fact, it must determine the length $h$ of the path from the routing, then its related position to the source. Supposing that $K$ and $K^{\prime}$ are known, node $i$ can calculate easily its corresponding $K(i, h)$ while maintaining an average $K$ in the path. Then, it informs the MAC layer about this $K(i, h)$ new value.

As an exterior observer point of view, the $K(i, h)$ values are attributed as follows: the middle node has the value $K$ (when $h$ is even, we attribute $K$ to the two middle nodes), and on its both sides, $K$ is decreased in the direction of the source and increased in the direction of the destination by a value of $K^{\prime}$ for each hop. On one side, whenever it is impossible to decrease $K$, the value of $K(i, h)$ maintains its last value. On the other side, $K(i, h)$ must maintain also the last value in a certain level so that we maintain an average of $K$.

For example, when $h=10, K=8$, and $K^{\prime}=2$, the set values of $K(i, h)$ is attributed to the set of nodes in $R_{s, d} \cup\{s\}$ starting from the source and ending with the node before the destination, that is, $\{2,2,4,6,8,8,10,12,14,14\}$. In this case, we have $K(4,10)=6, K(5,10)=8$, and so forth. We could also use another method to attribute values of $K(i, h)$. The key idea of the dynamic scheme is to attribute the values of $K(i, h)$ in an increasing manner starting from the source of a connection till the destination. For that, a simple algorithm can be turned on each node to determine its corresponding dynamic $K$ to a given route $R_{s, d}$.

\subsubsection{Analysis of the numerical results}

We draw some numerical results of the above formulas using the previous parameters $B=10$ and $K=8$ for the two cases $K^{\prime}=0$ and $K^{\prime}=2$. The maximum hop number $B=10$ allows about $2 * 10$ possible connections for each node. The forwarding probability is set to $f=0 \cdot 8$. The numerical results concern the average number of transmissions and stability for each node, then the end-to-end throughput and probability of success. We are also interested in evaluating the behavior of these, for each transmission probability, that is, when the channel suffer from low-to-high contention. All these performance metrics are shown from Figures 2 to 5. The main remarkable thing is that the stability and the throughput are considerably ameliorated with the dynamic scheme $\left(K^{\prime} \neq 0\right)$ compared to the static $K$ case $\left(K^{\prime}=0\right)$.

This remarkable amelioration is mainly due to the following facts.

(1) The dynamic scheme privileges the forwarded packets that come near the destination. It is better to encourage these packets to reach their destination, otherwise, the network will suffer more wasted bandwidth.

(2) The flow of packets from each source is limited on the first hops of each connection. If the network cannot support transporting more packets on a connection, it is better to limit the flow of new entering packets in the network. This is a load moderating issue.

As a consequence, Figure 2 shows that the average number of attempts in the dynamic scheme $\left(L\left(K^{\prime} \neq 0\right)\right)$ is always lower than the number of attempts in the static case, that is, $L\left(K^{\prime} \neq 0\right) \leq L\left(K^{\prime}=0\right)$. (Let $X\left(K^{\prime} \neq 0\right)$ and $X\left(K^{\prime}=0\right)$ 


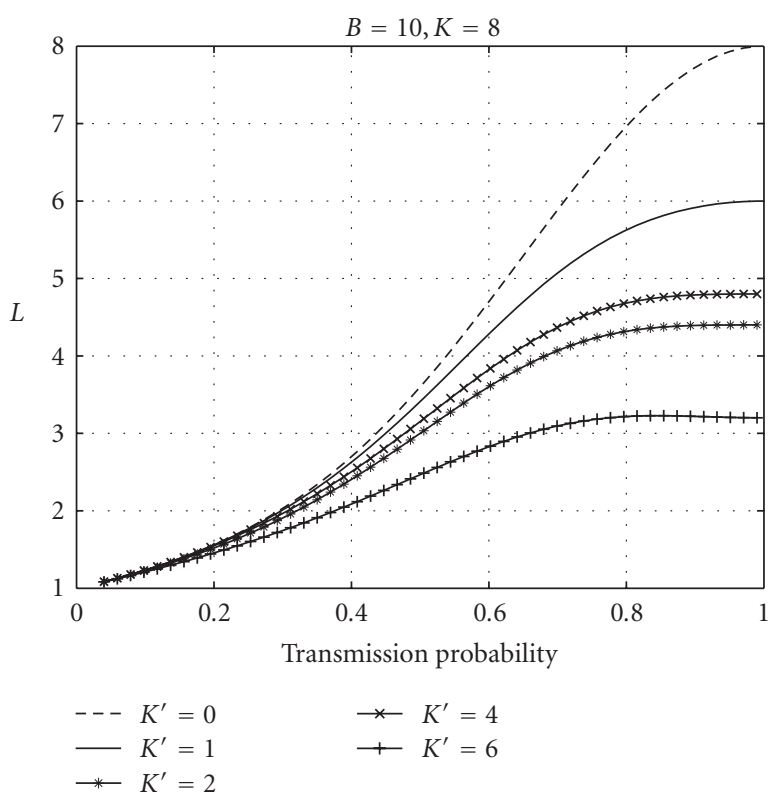

FIgURE 2: Average number of transmission $L$ for different $K^{\prime}$.

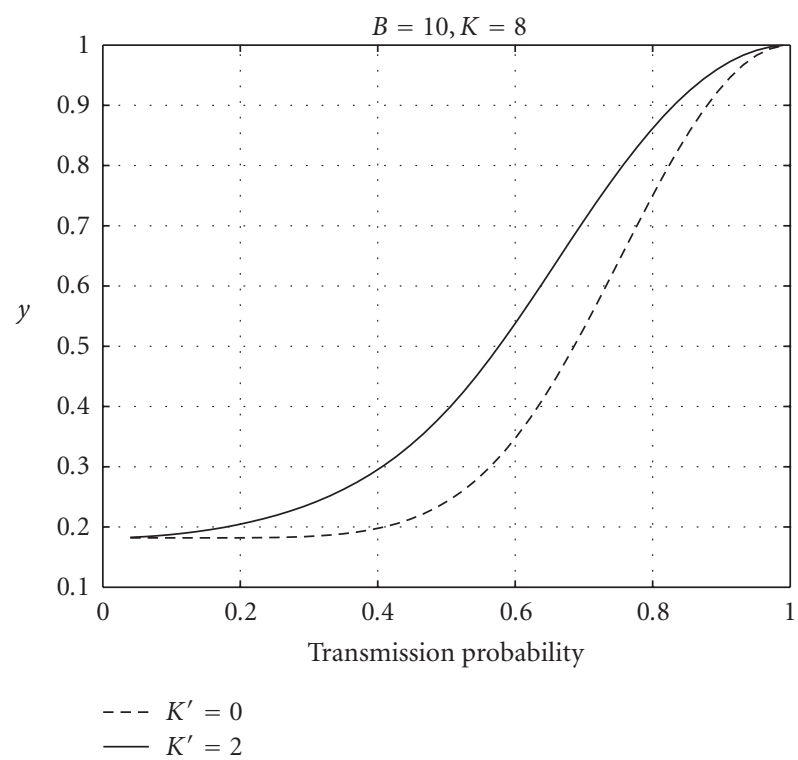

FIgURE 3: Stability region for the linear case with static and dynamic $K$.

be two symbols, where $X$ represents one of the parameters in the set $\{K, L, y, \overline{\text { thp }}, \overline{P \text { succ }}\}$ for the two dynamic and static $K$ case, resp.) It means that a packet needs less average number of retransmissions at each node throughout a path $R_{s, d}$ to be delivered on the destination, in the dynamic case. Therefore, the service rate of the forwarding packets will be faster. As shown in Figure 3, it has permitted a low load, and more new packets have entered the network, indicating a higher region of stability. For that, $y\left(K^{\prime} \neq 0\right) \geq y\left(K^{\prime}=0\right)$.

We distinguish two states of the network for two contention degrees when we use the dynamic scheme. These are shown on Figure 4 where for a given $P_{0}\left(P_{0} \simeq 0.55\right.$ in this

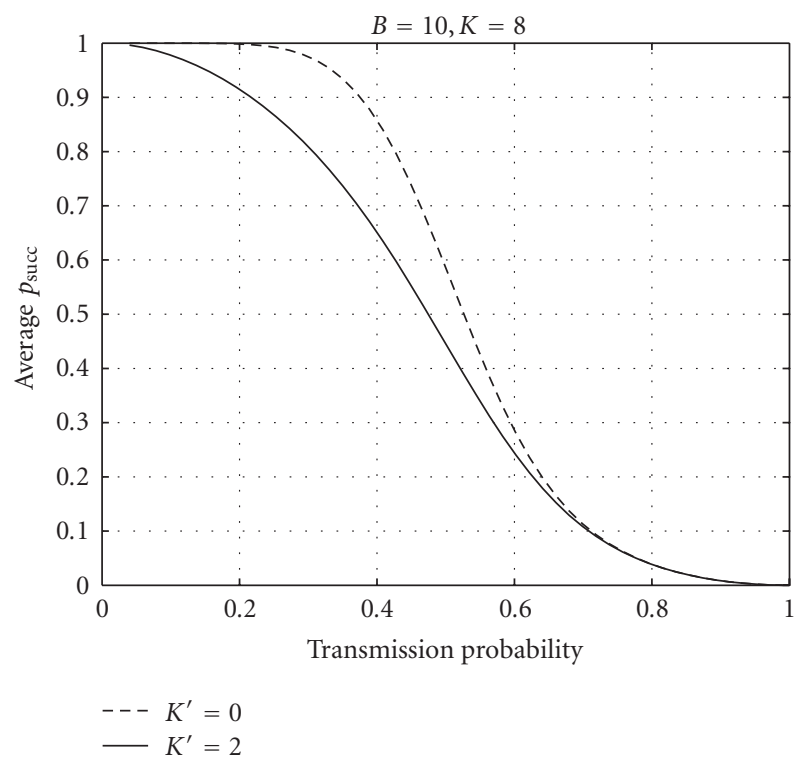

Figure 4: Average Probability of success for the linear case with static and dynamic $K$.

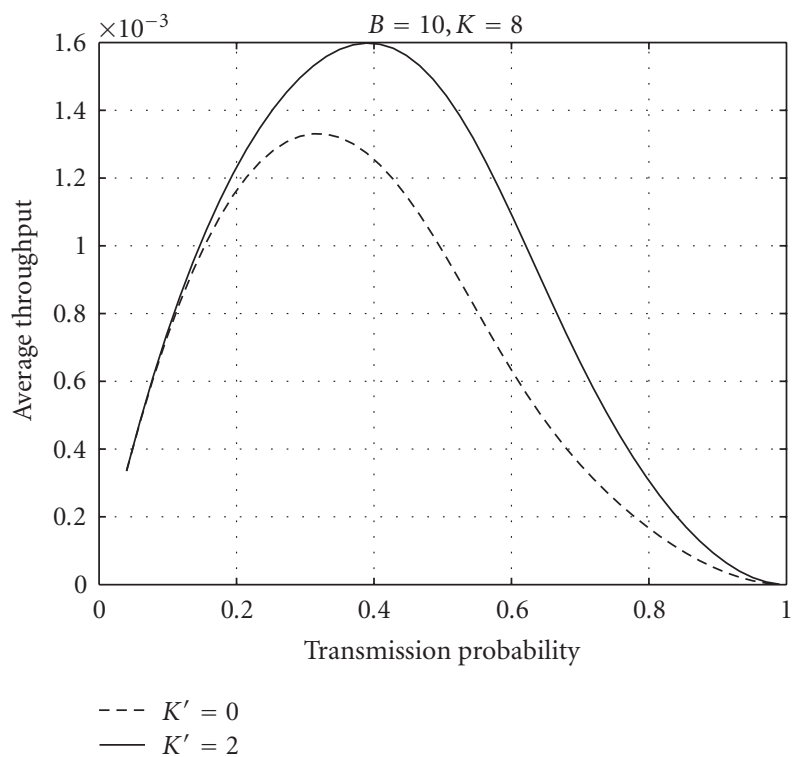

FIGURE 5: Average throughput for the linear case with static and dynamic $K$.

example), the probability of success for $K^{\prime} \neq 0$ comes near the one for $K^{\prime}=0$. Therefore, we distinguish the following.

(i) The severe state for a low-moderate contention: $P \leq P_{0}$ and $\overline{P \text { succ }}\left(K^{\prime} \neq 0\right)<\overline{P \text { succ }}\left(K^{\prime}=0\right)$. In this state, the nodes with $K\left(K^{\prime} \neq 0\right)<K$ have the aptitude to drop a lot of packets in the first nodes (near the source) on a path $R_{s, d}$, in such a manner that a less number of successful packets arrives to the destination compared to the static $K$ case. When dropping a lot of packets in the network, the load of each node diminishes, thus $y\left(K^{\prime} \neq 0\right) \geq y\left(K^{\prime}=0\right)$. Since the impact of the load in this state is higher than the end-to-end probability of success, the average throughput in the network becomes 
larger, that is, $\overline{\operatorname{thp}}\left(K^{\prime} \neq 0\right) \geq \overline{\operatorname{thp}}\left(K^{\prime} \neq 0\right)$, see Figure 5 . In low contention, we do not really need to limit a lot the retransmission of packets since they have a higher chance to reach the destination in the static $K$ case. The load control appears to be more efficient than controlling the success of packets to get a good throughput. In fact, giving more chance of success to packets approaching the destination is not the main reason of amelioration in low-channel contention case. Instead, decreasing the limit number of retransmissions of source packets is more important as it has the impact on the load of forwarding queues.

(ii) The moderate state for a moderate-high contention: $P \geq P_{0}$ and $\overline{P \operatorname{succ}}\left(K^{\prime} \neq 0\right) \simeq \overline{P \text { succ }}\left(K^{\prime}=0\right)$. In this state, dropping packets with $K\left(K^{\prime} \neq 0\right)<K$ is more efficient than the severe state, in such a manner that even the end-to-end probability of success comes closer to the static case. In high contention, a packet that has traveled through many nodes in the static $K$ case is more susceptible to drop. When dropping such packet, we loose all retransmission slots from the source until its drop. Therefore, increasing $K$ behind the middle node on a path $R_{s, d}$ helps conserving the packets from drop and minimizes the number of loosed slots. In this way, the throughput and the load are ameliorated as shown in Figures 5 and 3, respectively. This also can be verified with Figure 2, where $L\left(K^{\prime} \neq 0\right) \leq L\left(K^{\prime}=0\right)$.

These two states can merge to one state for some values of $K, K^{\prime}$, or $B$. However, the dynamic scheme maintains its advantage compared to the static $K$ case.

\subsubsection{Impact of $B, K$, and $K^{\prime}$}

We study the impact of $B, K$, and $K^{\prime}$ on the performance of the network. We proceed by comparing the static and the dynamic case. We show on the following figures the gain ratio while using the dynamic case in terms of these last parameters. The gain ratio of the static-dynamic $K$ for the average throughput and the stability region, presented in Figures 7 to 10 , is defined as $\left(X\left(K^{\prime} \neq 0\right)-X\left(K^{\prime}=\right.\right.$ $0)) / X\left(K^{\prime}=0\right)$, where $X$ is the average of thp or $y$ for all $P$. From these figures, the gain ratio of the average throughput and the stability region evolve in a similar way when varying $B, K$, or $K^{\prime}$. Remark that the average throughput is mainly affected by the load variation of each node from (7).

(1) Impact of $B$. Let $\Delta L$ be the difference between the average number of transmissions for $K^{\prime}=0$ and $K^{\prime} \neq 0$, that is, $\Delta L=L\left(K^{\prime}=0\right)-L\left(K^{\prime} \neq 0\right) . \Delta L$ is an increasing function with $B$ as shown in Figure 6 which verifies the increasing gain ratio of the Figure 7 . The dynamic scheme has a good performance in large multihop networks.

(2) Impact of $K$. When $K$ tends toward large values, the two dynamic and static cases tend to each other, that is, we tend to zero gain ratio. So, each packet will be retransmitted until achieving a success. It does neutralize our dynamic scheme. Also, the impact of $K$ depends on $K^{\prime}$ as we see in Figures 8 and 9 . For some $K$ near the value of $K^{\prime}$ or multiple of it, we have picks of gain ratio that decrease with $K$. For $K=5$, we have the maximum possible gain in these two figures. It is a question of how we use $K^{\prime}$ according to $K$ so that the dynamic scheme operates in an optimal point.

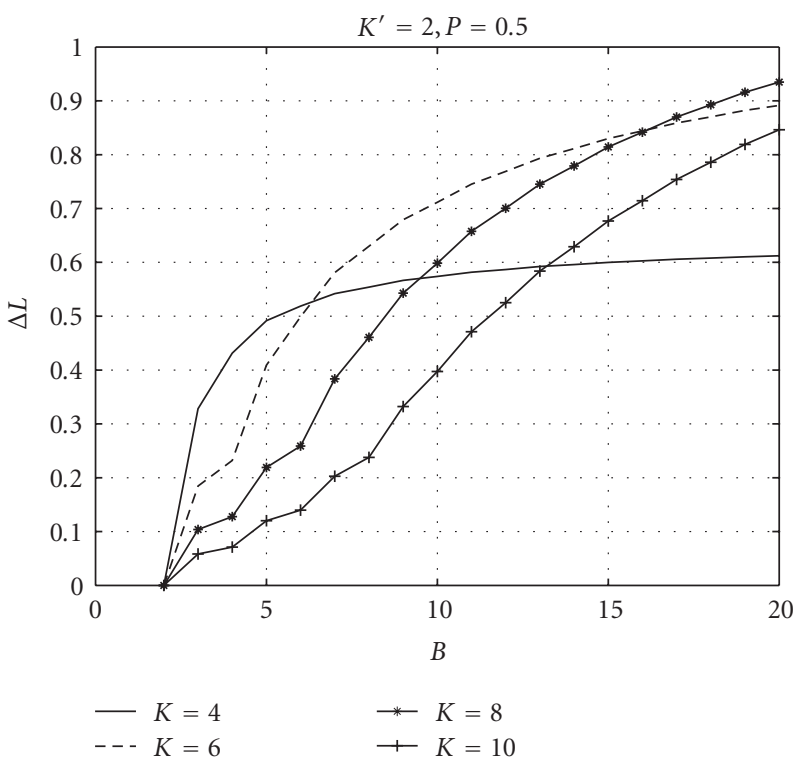

Figure 6: $\Delta L$ versus $B$ for different $K$, for $K^{\prime}=2$ and $P=0.5$.

In fact, for some values of $K$ and $K^{\prime}$, the source nodes (and the nearest nodes to the source) may be affected by very low dynamic $K$ or not (according to the scheme calculation of dynamic $K$ ). This is why we can see picks of gain or not in Figures 8 and 9 . For a fixed $K^{\prime}$ and $P$, we plot the quantity $\Delta L$ as a function of $K$ in Figure 6. We observe that for small $B, \Delta L$ decreases with $K$, so in Figure 8, the gain ratio is also decreasing. Remark that Figure 8 (as Figures 7, 9, and 10) presents the gain ratio for all $P$, whereas Figure 6 is presented for a fixed $P=0.5$. Therefore, for $P=0.5$ and large $B$, our scheme obtains high performance with higher $K$. For that, the impact of $B$ is considerable compared to $K$.

(3) Impact of $K^{\prime}$. The dynamic scheme is defined for $K^{\prime} \leq K$. From Figure 10, choosing $K^{\prime}=7$ for $K=8$ causes severe drops at the beginning of a path since $K\left(K^{\prime} \neq 0\right)=1$ for $K\left(K^{\prime} \neq 0\right)<K$. Even though the gain ratio is clearly large. The difference between $K^{\prime}=2$ and $K^{\prime}=4$ in Figure 10 is explained by Figure 2 where $L\left(K^{\prime}=2\right)<L\left(K^{\prime}=4\right)$.

\subsection{Asymmetric network case}

Consider an asymmetric static wireless network with 11 nodes as shown in Figure 11. We choose the following parameters: $f_{i} \equiv f=0.8$ and $P_{i}$ in a manner of enabling stability, for all $i$. Let $P_{2}=P_{3}=P_{7}=P_{8}=0.3, P_{4}=P_{10}=$ $0.4, P_{5}=0.5$ be the fixed transmission probabilities for nodes $2,3,4,5,7,8$, and 10 while $P_{i} \equiv P$ for all other $i$. Many nodes need to have a fix transmission probabilities so to get stable queues for all nodes. The default maximum number of transmissions is $K$. In the static case, $K_{i, s, d} \equiv K$, while in dynamic case, $K_{i, s, d}$ is chosen using the dynamic scheme presented previously (Sections 3 and 4.1). We implement this dynamic scheme in our discrete time simulator, so we can evaluate performance and validate numerical results with simulations. 
TABLE 1: Values of the dynamic maximum number of transmissions for $K^{\prime}=1$ and $K=4$.

\begin{tabular}{llllllllll}
\hline Nodes & 2 & 3 & 4 & 5 & 6 & 7 & 8 & 10 \\
\hline Conn. $a$ & 0 & 0 & 3 & 4 & 0 & 5 & 0 & 0 \\
Conn. $b$ & 4 & 3 & 5 & 0 & 0 & 0 & 0 & 0 \\
Conn. $c$ & 0 & 0 & 0 & 0 & 0 & 0 & 5 & 4 \\
Conn. $d$ & 0 & 5 & 0 & 4 & 0 & 0 & 3 & 0 \\
Conn. $e$ & 0 & 0 & 4 & 5 & 3 & 0 & 0 & 0 \\
\hline
\end{tabular}

TABle 2: Values of the dynamic maximum number of transmissions for $K^{\prime}=2$ and $K=4$.

\begin{tabular}{llllllllll}
\hline Nodes & 2 & 3 & 4 & 5 & 6 & 7 & 8 & 10 \\
\hline Conn. $a$ & 0 & 0 & 2 & 4 & 0 & 6 & 0 & 0 \\
Conn. $b$ & 4 & 2 & 6 & 0 & 0 & 0 & 0 & 0 \\
Conn. $c$ & 0 & 0 & 0 & 0 & 0 & 0 & 6 & 4 \\
Conn. $d$ & 0 & 6 & 0 & 4 & 0 & 0 & 2 & 0 \\
Conn. $e$ & 0 & 0 & 4 & 6 & 2 & 0 & 0 & 0 \\
\hline
\end{tabular}

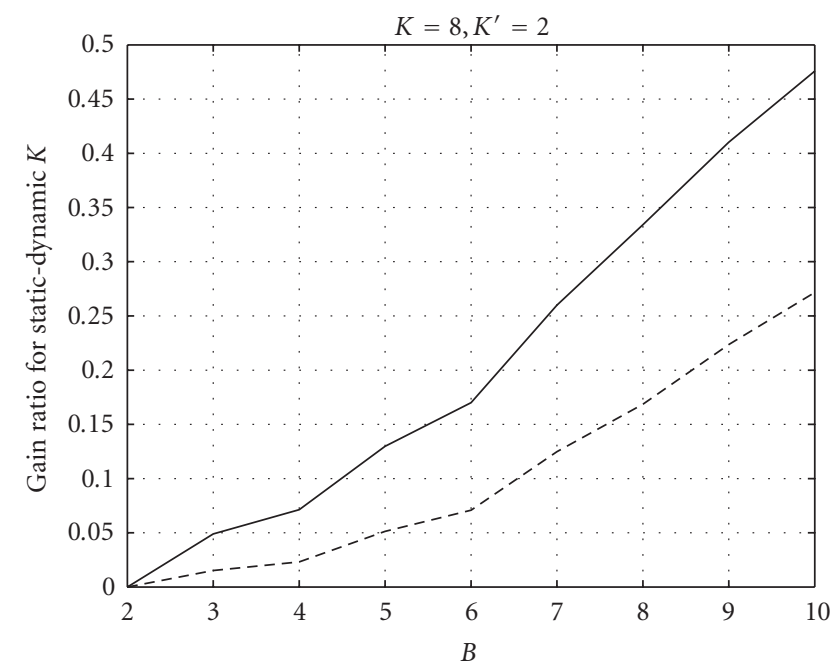

- Average throughput

$$
---y
$$

FIgURE 7: Gain ratio of dynamic case compared to the static one versus $B$ (linear network).

\subsubsection{A first numerical and simulation study}

Let $a, b, c, d$, and $e$ be the five connections established on the network of Figure 11, as indicated in the same figure. We compare from Figures 12 to 16 the dynamic ( $K=4$ and $\left.K^{\prime}=1\right)$ and the static $\left(K=4\right.$ and $\left.K^{\prime}=0\right)$ cases. Note that $y_{j}=1$ for $j \in\{1,6,9,11\}$, since these nodes do not forward packets. Firstly, the forwarding source nodes $(3,4$, and 8$)$ of the connections $a, b$, and $d$ have affected small dynamic $K$ for their new packets (packets from Q): $K_{3,3,6}=K_{4,4,11}=$ $K_{8,8,2}=3$ and higher dynamic $K$ for their forwarding packets: $K_{3,8,2}=K_{4,3,2}=K_{8,11,6}=5$ and $K_{4,6,7}=4$ (see Table 1 for $K^{\prime}=1$ and Table 2 for $K^{\prime}=2$ ). Note that "Conn." means "connection." In fact, there are two advantages on giving smaller $K$ for new packets. The first one is that it

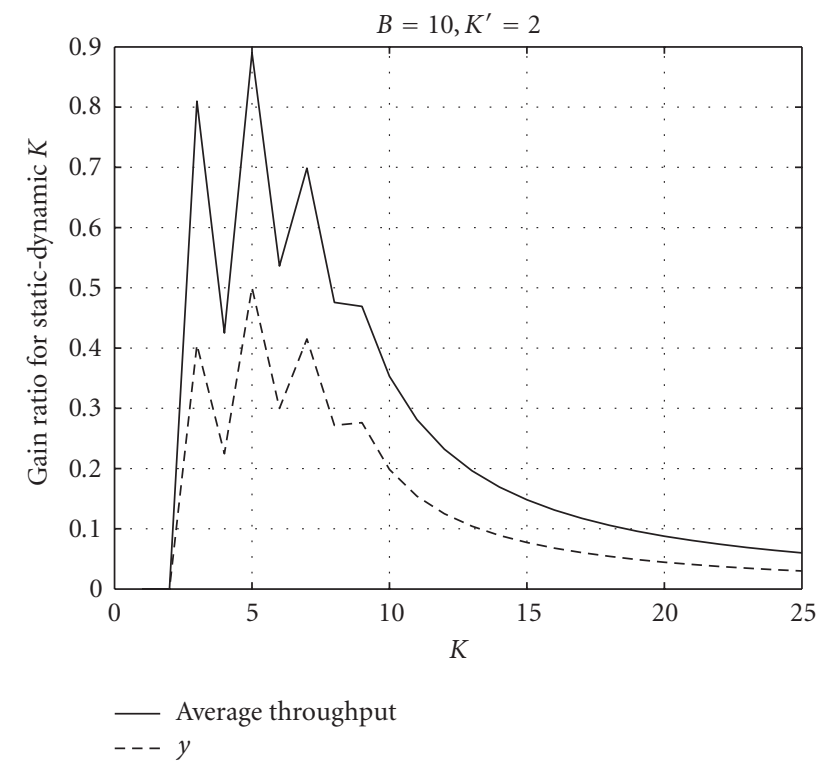

FIgURE 8: Gain ratio of dynamic case compared to the static one versus $K$ for $K^{\prime}=2$ (linear network).

gives more priority to the forwarding queue. Therefore, we observe less load, then more aptitude to send new packets. The second one is that it can optimize the load on the nodes belonging to the source connection, in a saturated network case. In fact, it can make severe drops of new packets (due to small $K$ ) that can diminish the flow of packets in a connection and maintain necessary packets. Figure 12 supports our comment: the stability region or the aptitude of sending new packets is considerably ameliorated in nodes 3,4 , and 8 . In addition, the throughput of connection $d$ has beneficed from the increase of $y_{8}$ of its source node 8 , while the two others $a$ and $b$ have maintained their throughput approximatively unchanged.

Secondly, as the nodes 6 and 11 do not forward packets in our example, then $y_{6}=y_{11}=1$. These two nodes are the 


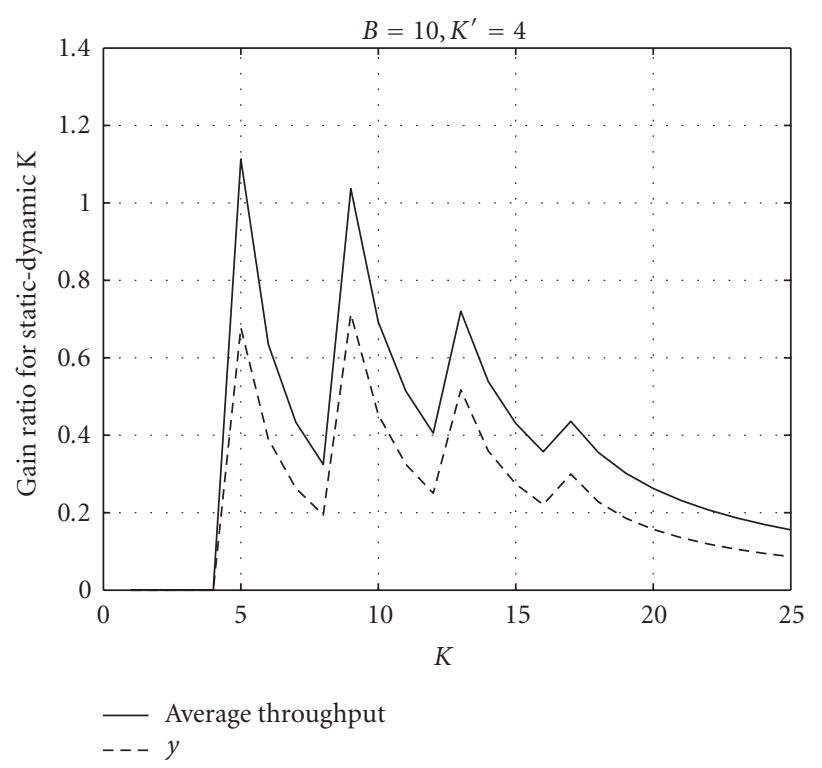

Figure 9: Gain ratio of dynamic case compared to the static one versus $K$ for $K^{\prime}=4$ (linear network).

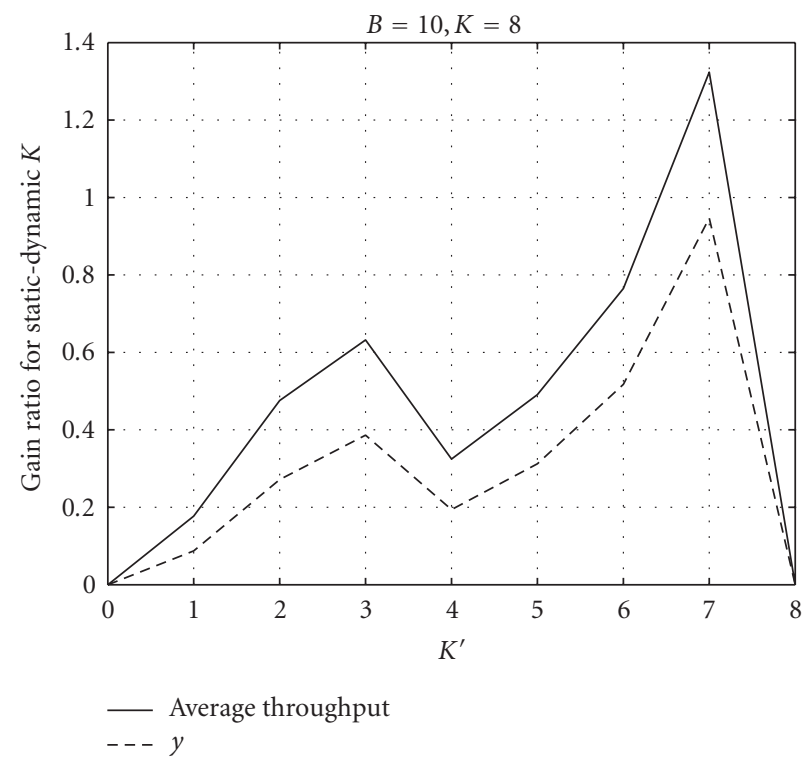

FIgURE 10: Gain ratio of dynamic case compared to the static one versus $K^{\prime}$ (linear network).

source of connections $e$ and $c$, respectively. From Figure 14, the throughput of these two connections are clearly higher in the dynamic scheme and do not depend on any forwarding queue loads in these connections. However, the packets sent from nodes 6 and 11 are limited by the fact that $K_{6,6,7}=$ $K_{11,11,6}=3$, but there are privileged at nodes 5 and 8 with $K_{5,6,7}=K_{8,11,6}=5$. Each drop at these latter nodes is more expensive than the drop at the source nodes (as explained previously). For that, the throughput of these connections (see Figure 14) was only affected by the end-to-end success of packets in an interval of time without the impact of $y_{6}$ and $y_{11}$ which are the source aptitudes of sending new packets.

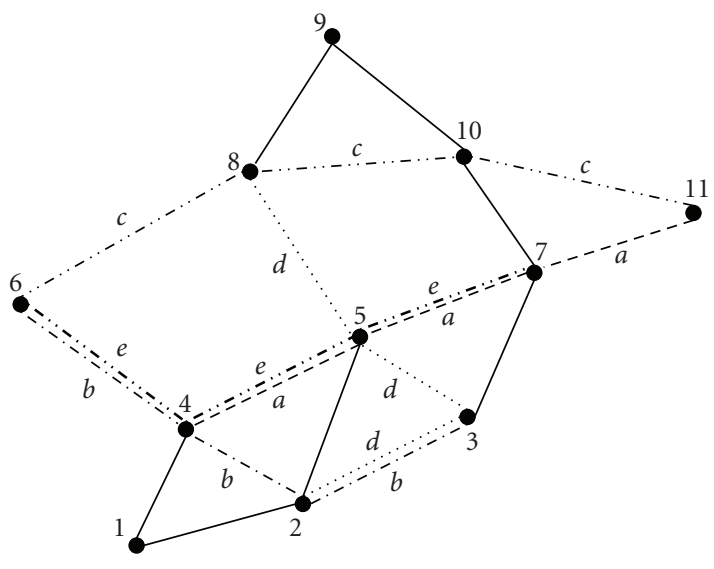

Connection $a$ : 4-5-7-11

Connection $b$ : 3-2-4-6

Connection $c$ : 11-10-8-6

Connection $d: 8-5-3-2$

Connection $e: 6-4-5-7$

Figure 11: Ad hoc wireless network.

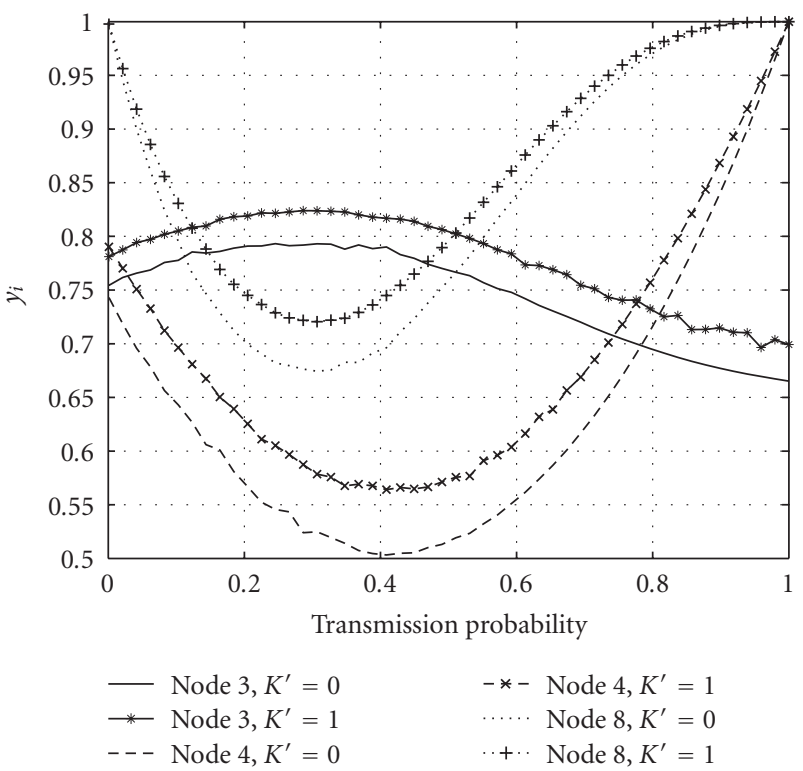

FIgURE 12: Stability region $y_{i}$ of the dynamic $\left(K^{\prime}=1\right)$ and static $\left(K^{\prime}=0\right)$ cases for $K=4$ (nodes 3,4 , and 8 of the asymmetric network of Figure 11).

Note that nodes 2, 5, 7, and 10 have not really changed their region stability with the dynamic scheme in this example (see Figure 13).

Thirdly, the end-to-end delay of a connection gives a global vision on the stability of nodes that forms this connection. Precisely, it is mainly affected by the waiting time on the forwarding intermediate queues. In Figure 15, the high delay of connection $c$ clearly reflects the high and moderate charges of nodes 10 and 8 shown in Figures 13 and 12, respectively. In Figure 16, connection $a$ has maintained 


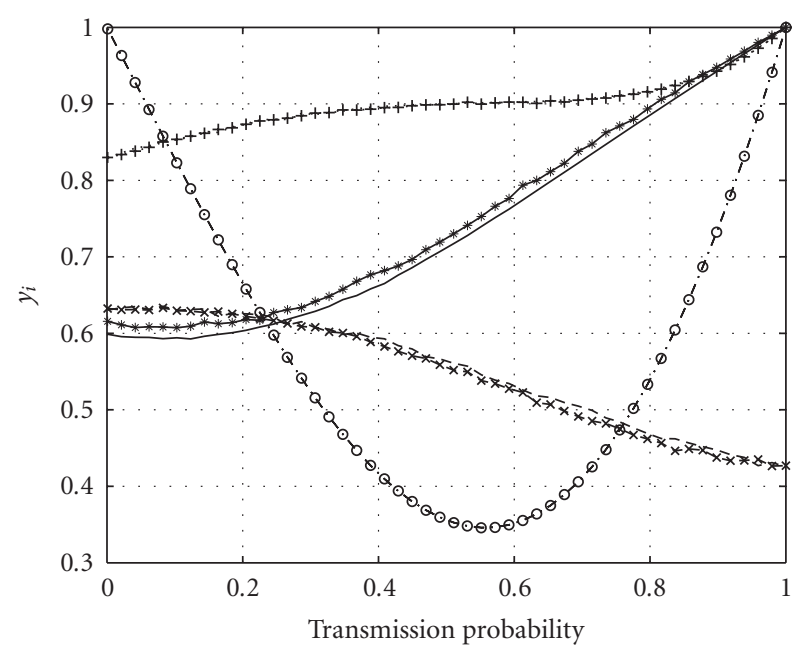

$$
\begin{array}{lll}
\text { - Node } 2, K^{\prime}=0 & \cdots \cdots & \text { Node } 7, K^{\prime}=0 \\
\text { * Node } 2, K^{\prime}=1 & \cdots+\cdot \text { Node } 7, K^{\prime}=1 \\
-- \text { Node } 5, K^{\prime}=0 & -\cdots \text { Node } 10, K^{\prime}=0 \\
-*-\text { Node } 5, K^{\prime}=1 & - \text { - } & \text { Node } 10, K^{\prime}=1
\end{array}
$$

FIgURE 13: Stability region $y_{i}$ of the dynamic $\left(K^{\prime}=1\right)$ and static $\left(K^{\prime}=0\right)$ cases for $K=4$ (nodes $2,5,7$, and 10 of the asymmetric network of Figure 11).

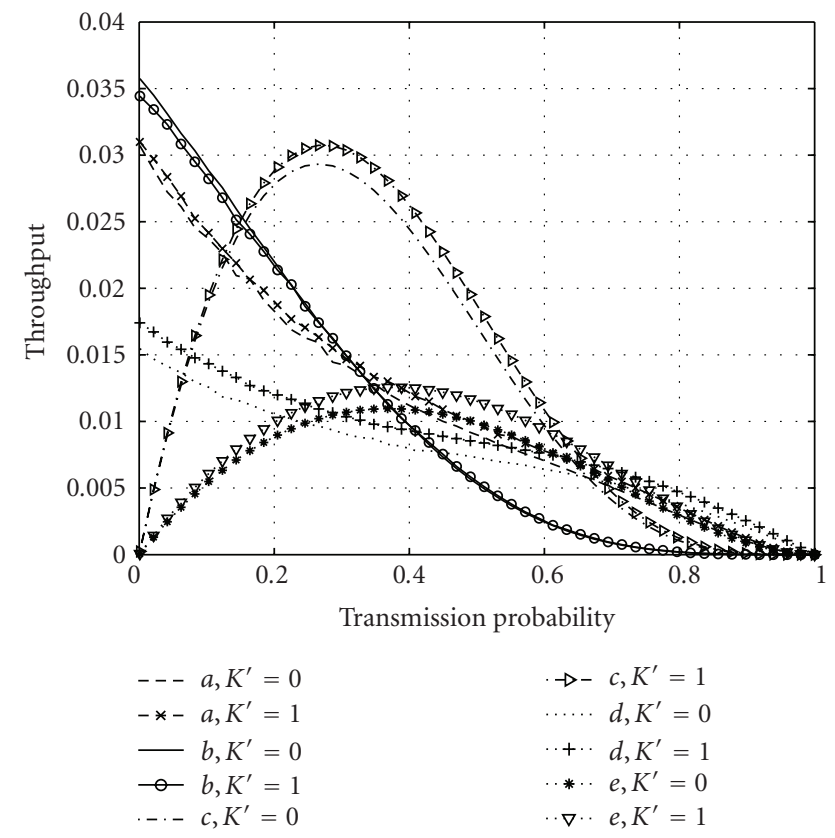

Figure 14: Throughput of the dynamic $\left(K^{\prime}=1\right)$ and static $\left(K^{\prime}=0\right)$ cases for $K=4$ (asymmetric network of Figure 11).

the same delay in the dynamic as in the static case. This was a consequence of the $y_{5}$ and $y_{7}$ unchanging (not so changed) in these two cases. Delay of connections $b, c$, and $e$ have been ameliorated in the dynamic case due to the forwarding aptitude of source nodes 4 and 8 that belong to these connections.

What about the gain percentages of our scheme compared to the static one? On one hand, we can observe from

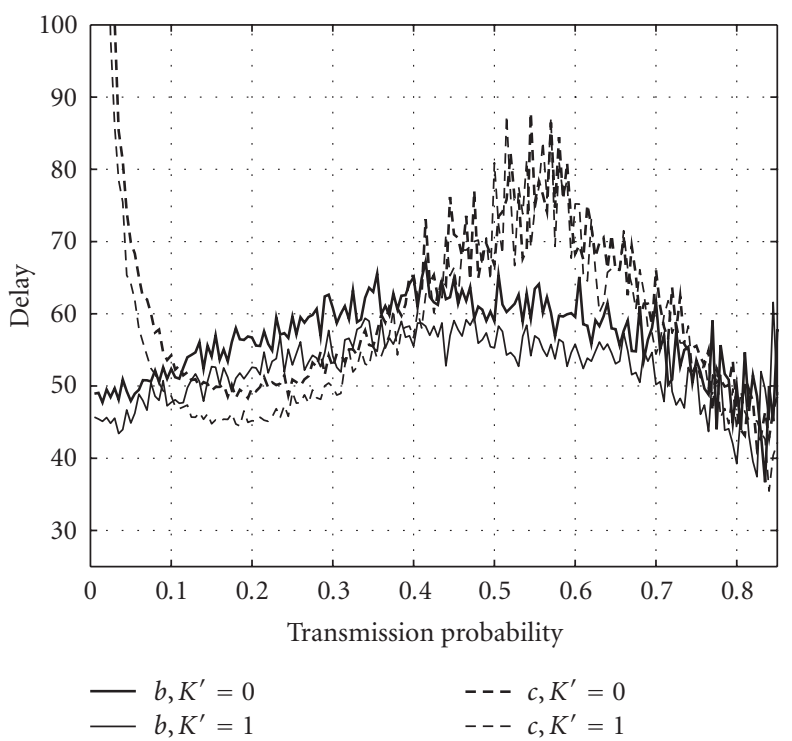

Figure 15: Delay of the dynamic $\left(K^{\prime}=1\right)$ and static $\left(K^{\prime}=0\right)$ cases for $K=4$ (connections $b$ and $c$ of the asymmetric network of Figure 11).

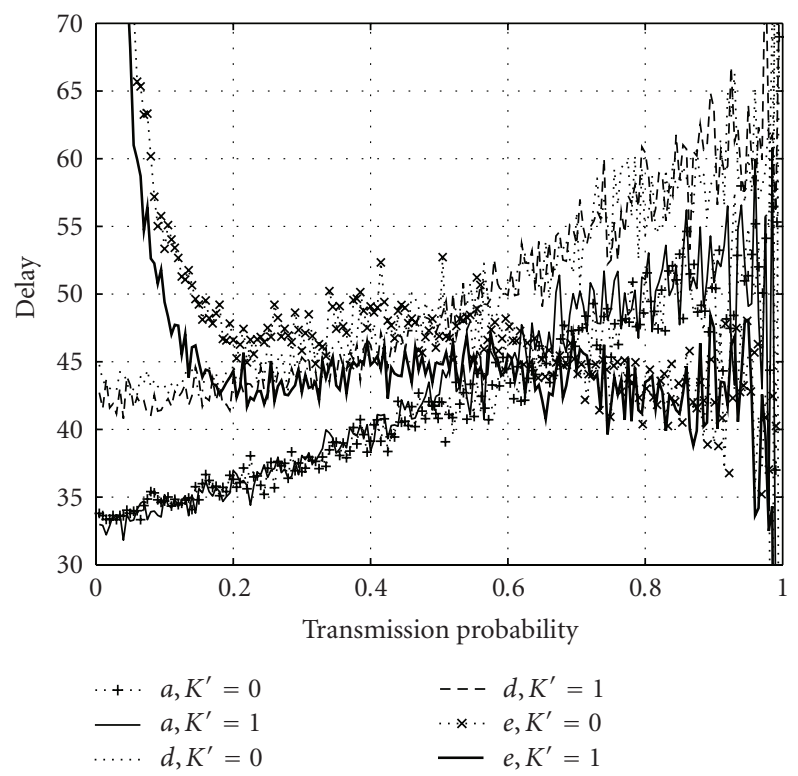

FIgURE 16: Delay of the dynamic $\left(K^{\prime}=1\right)$ and static $\left(K^{\prime}=0\right)$ cases for $K=4$ (connections $a, d$, and $e$ of the asymmetric network of Figure 11).

the presented Figures 14, 15, and 16 for $K^{\prime}=1$ that the gain varies in function of the probability of transmission. Therefore, for a $P=0.4$, the throughput amelioration reaches $20 \%$ for connection $d$ and is around 9\% for connections $c$ and $e$. The delay for this same $P$ is around $15 \%$ for connection $b$ and around $11 \%$ for connections $c$ and $e$. On the other hand, Figures 17 and 18 can show a gain up to $50 \%$ for the throughput and up to $30 \%$ for the delay, for 


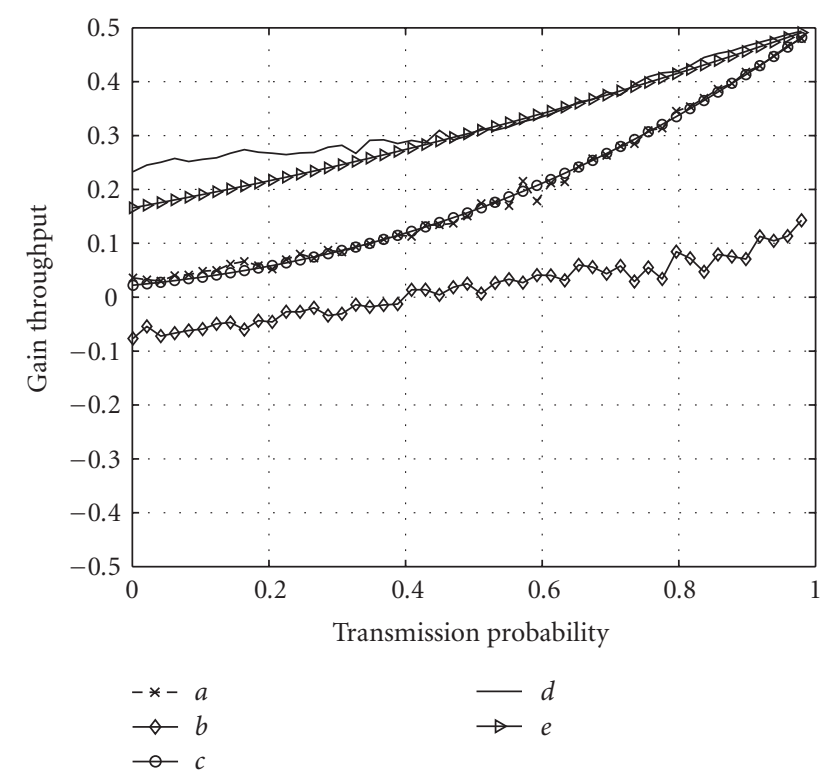

Figure 17: Throughput of the dynamic $\left(K^{\prime}=2\right)$ and static $\left(K^{\prime}=0\right)$ cases for $K=4$ (asymmetric network of Figure 11).

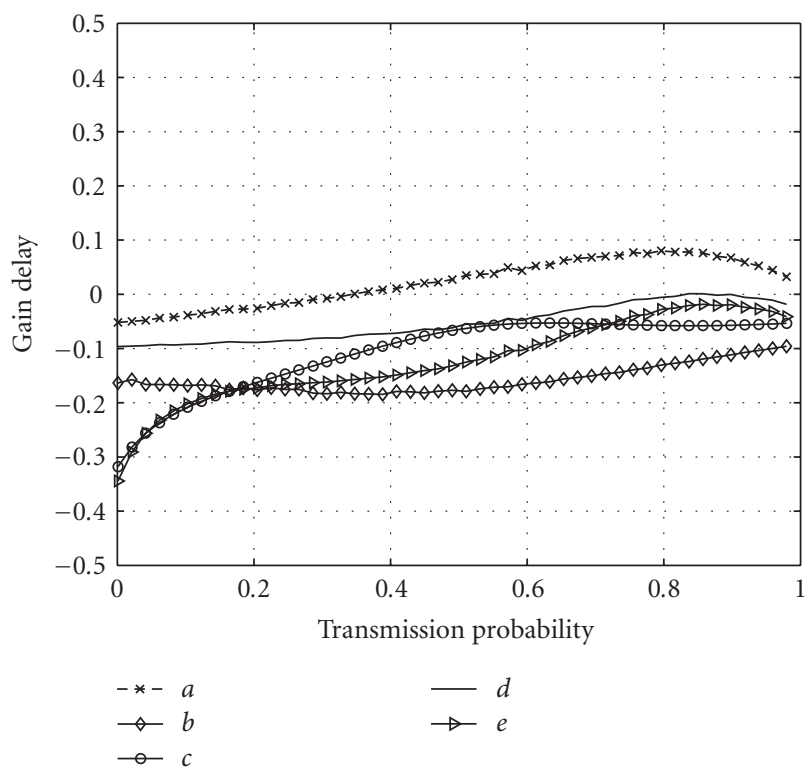

FIgURe 18: Delay of the dynamic $\left(K^{\prime}=2\right)$ and static $\left(K^{\prime}=0\right)$ cases for $K=4$ (asymmetric network of Figure 11).

$K^{\prime}=2$. In the following paragraph, we will discuss some issues from these figures.

\subsubsection{Discussion}

Here, we start our discussion on the observations of previous figures of the asymmetric network. In summary, the dynamic scheme has better performances when some of the source nodes collaborate by forwarding packets and when these

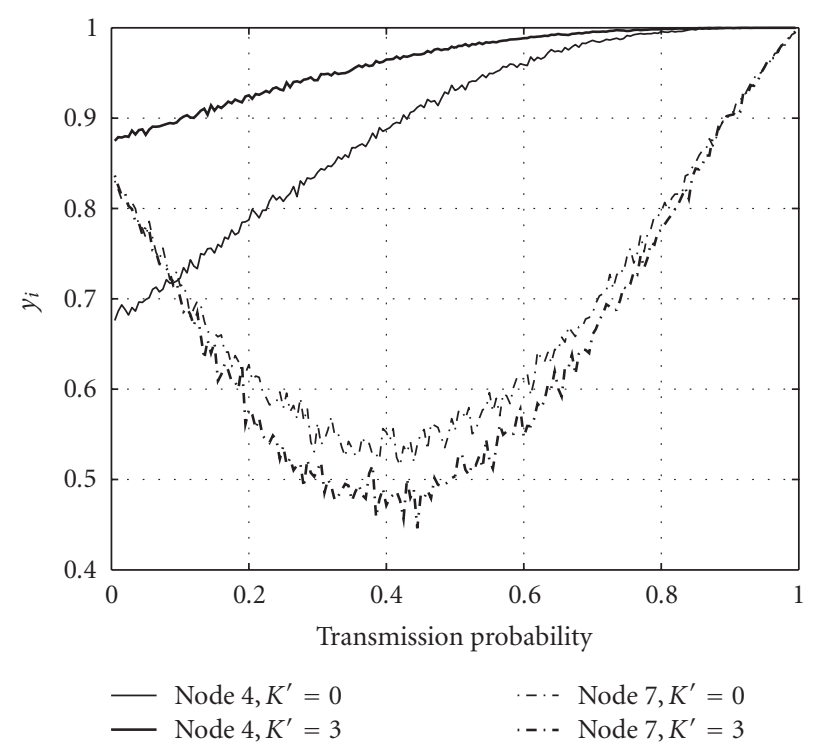

FIGURE 19: Stability region $y_{i}$ of the dynamic $\left(K^{\prime}=3\right)$ and static $\left(K^{\prime}=0\right)$ cases for $K=4$ (asymmetric network of Figure 11).

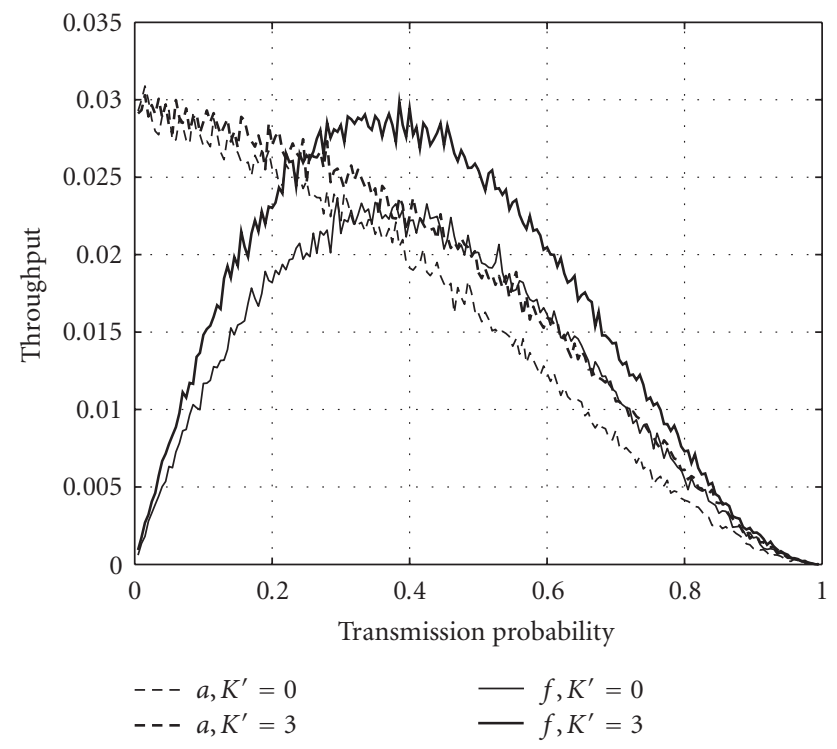

Figure 20: Throughput of the dynamic $\left(K^{\prime}=3\right)$ and static $\left(K^{\prime}=0\right)$ cases for $K=4$ (asymmetric network of Figure 11).

kinds of sources are well distributed in the network. In fact, there are two properties that help a connection to get a good performance:

(1) a connection must include within its intermediate nodes a source node of another connection;

(2) the source node of a connection must not forward packets.

The first one ameliorates the delay and the second one the throughput. These two properties are jointly found in 


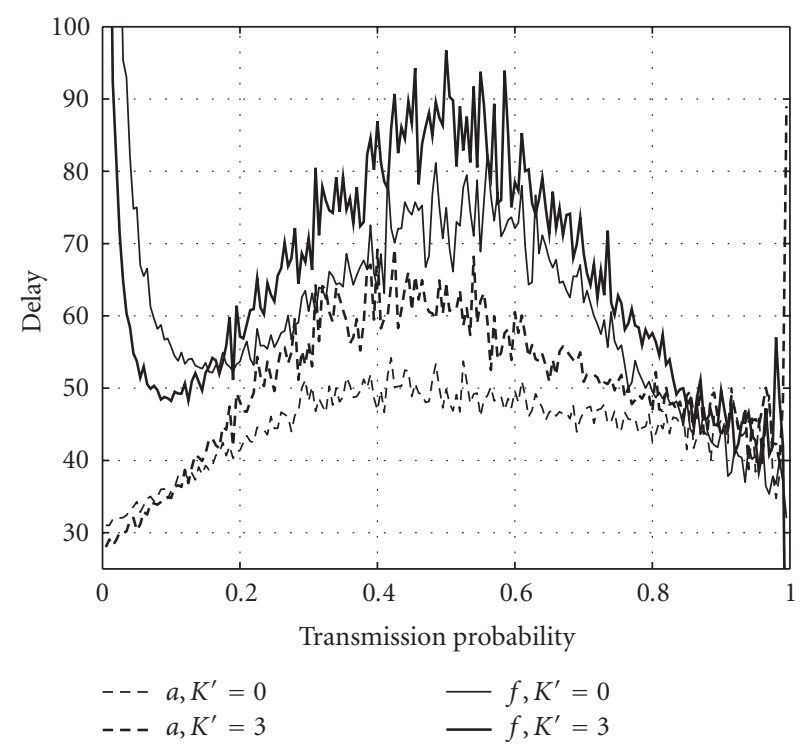

Figure 21: Delay of the dynamic $\left(K^{\prime}=3\right)$ and static $\left(K^{\prime}=0\right)$ cases for $K=4$ (asymmetric network of Figure 11).

connections $c$ and $e$. Furthermore, the good performance (of throughput and delay) of connection $c$ and $e$ confirms this conclusion. In fact, nodes 11 and 6 are two nodes that do not forward packets (see network of Figure 11) and in addition they are sources of the connections $c$ and $e$, respectively. Therefore, the traffic intensity from these two nodes to their corresponding connections is maximal since no forwarding packets are to be transmitted. This is why we can expect a good throughput. Furthermore, the set of intermediate nodes of connections $c$ and $e$ contains a source node of other connections: node 8 for connection $c$ and node 4 for connection $e$. These latter source nodes experience less delay due to the fact that they have been affected lower limit number of retransmissions. Consequently, the end-to-end delay of connections $c$ and $e$ would be lower.

When one of these two properties is not found, then three situations are presented: a connection of the dynamic scheme

(1) maintains unchanged one performance criterion (throughput or delay) and ameliorates the other one;

(2) deteriorates one performance criterion and ameliorates the other one;

(3) maintains unchanged the performance.

These three situations are with comparison to the static case. An example of the first and the second situation is the connections $a, b$, and $d$ in Figures 17 and 18 depending on the transmission probability. In the second situation, the new scheme does not overcome the existing tradeoff throughput delay for a given connection, but it enables a benefit to other connections, and thus the aggregate performance of the network is ameliorated. To overcome this tradeoff of some given connections of this second situation, we have introduced to the new dynamic scheme the capability to reset

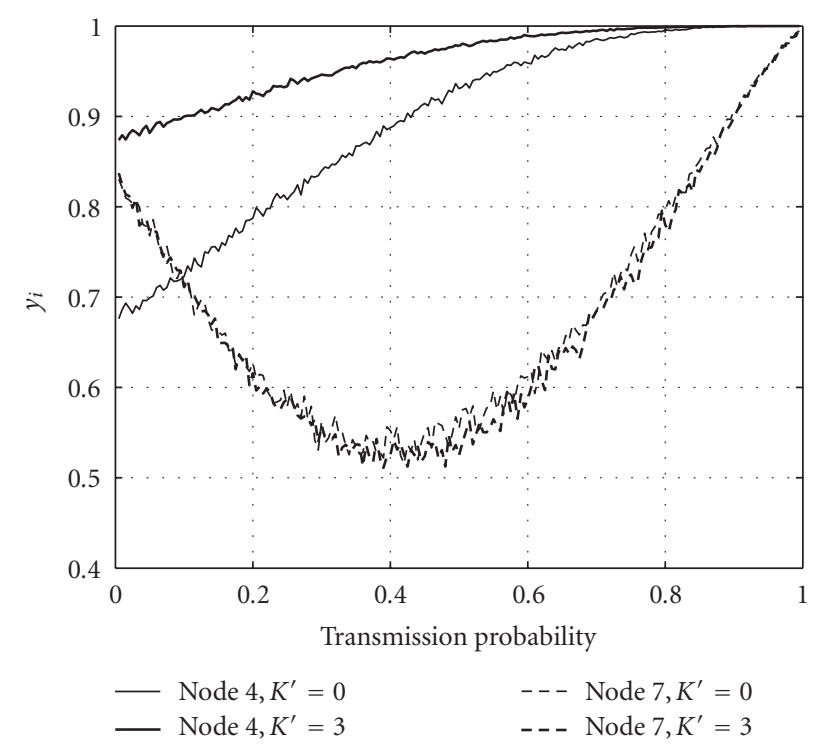

Figure 22: Stability region $y_{i}$ of the dynamic $\left(K^{\prime}=3\right)$ and static $\left(K^{\prime}=0\right)$ cases for $K=4$ with a reset of the $K$ value of node 7 (asymmetric network of Figure 11).

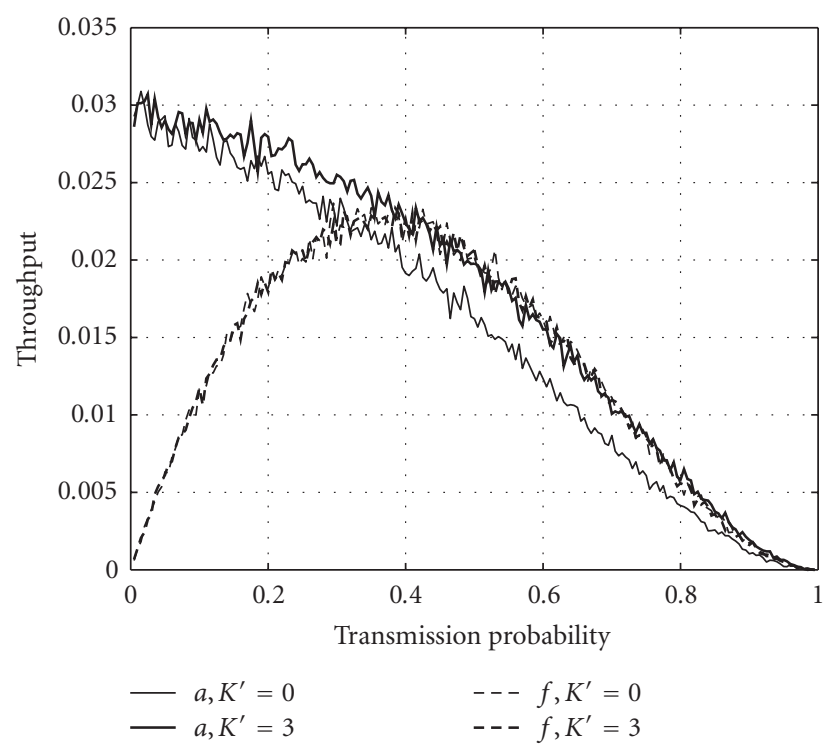

FIGURE 23: Throughput of the dynamic $\left(K^{\prime}=3\right)$ and static $\left(K^{\prime}=0\right)$ cases for $K=4$ with a reset of $K\left(K^{\prime}=3\right)$ on node 7 of connection $f$ (asymmetric network of Figure 11).

the dynamic value of $K$, that is, to reallocate the default value $K$ instead of the dynamic value only on the case where $K\left(K^{\prime} \neq 0\right)>K\left(K^{\prime}=0\right)$. We study this option of reallocating $K$ in the next section and we call it the reset technique.

\subsubsection{A second simulation study with the reset technique}

Here, we consider only three connections $a, b$, and $f$, where $f$ is formed by the successive nodes $9-10-7-3$ with node 9 as a source and node 3 as a destination. $a$ and $b$ are the 


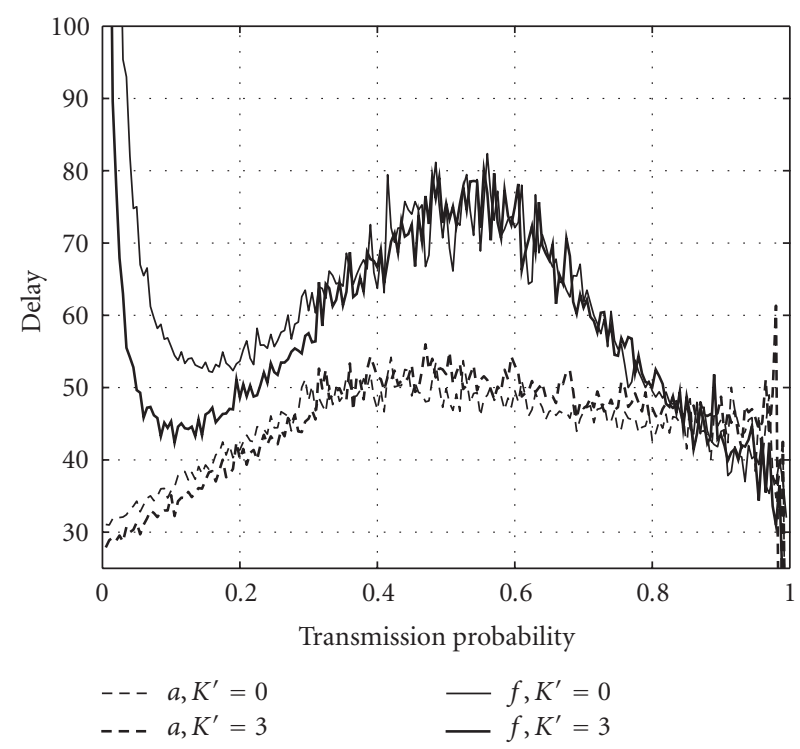

Figure 24: Delay of the dynamic $\left(K^{\prime}=3\right)$ and static $\left(K^{\prime}=0\right)$ cases for $K=4$ with a reset of $K\left(K^{\prime}=3\right)$ on node 7 of connection $f$ (asymmetric network of Figure 11).

same as previously mentioned. Connection $b$ causes node 4 to forward. The default value of $K$ is maintained to 4 . But, we choose $K^{\prime}=3$. In this manner, the value of $K\left(K^{\prime}=3\right)$ for node 7 is set to 7 for the two connections $a$ and $f$. Therefore, the stability of node 7 becomes critical and is penalized when the contention on the channel increases: many retransmissions of each single packet causes more additional waiting time on the forwarding queue. So the choice of high $K\left(K^{\prime}=3\right)$ on this node is not really judicious for itself as shown in Figure 19: $y_{7}\left(K^{\prime}=3\right)<y_{7}\left(K^{\prime}=0\right)$, nor for the connection delays as shown in Figure 21. However, a considerable amelioration on the throughput is clearly observed in Figure 20.

These observations correspond to the second situation described previously. Each node suffering from a degradation of stability appeals the reset technique. In fact, each node uses the following three steps to test and apply if needed, the reset technique as follows.

(1) If the average queue size exceeds a given threshold, then go to the second step, else do nothing. The average waiting time in the queue can be another criteria to decide whether to use the reset technique or not. It is directly related to the queue size. Also, the estimation of $y\left(K^{\prime}=0\right)$ and the measurement of $y\left(K^{\prime} \neq 0\right)$ can also be used for the decision.

(2) Choose judiciously a connection (according to its data type and if it is not yet chosen) between those traversing it.

(3) Apply the reset of $K\left(K^{\prime} \neq 0\right)$ to the default value $K$, that is, set $K\left(K^{\prime} \neq 0\right) \equiv K$ for the connection chosen in step 2.

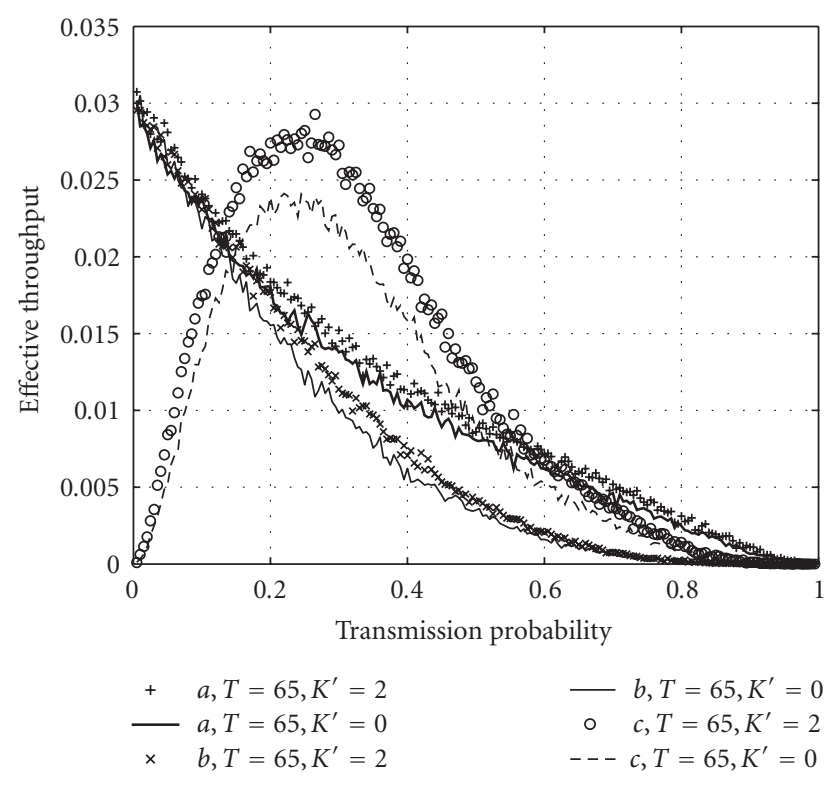

FIgURE 25: The effective throughput of the dynamic $\left(K^{\prime}=2\right)$ and the static $\left(K^{\prime}=0\right)$ cases for $K=4$ and $T=65$ (connections $a, b$, and $c$ of the asymmetric network of Figure 11).

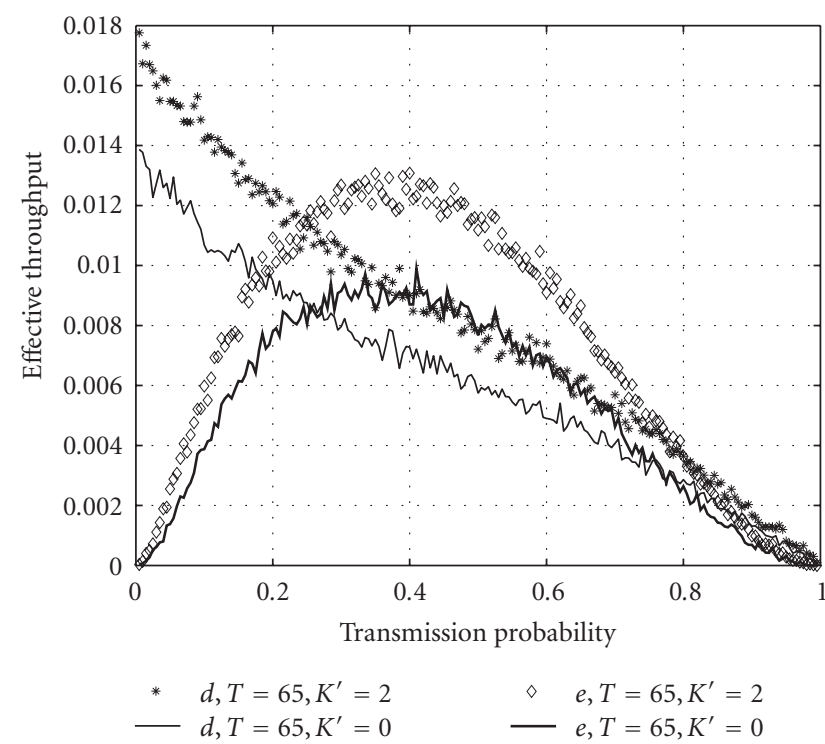

Figure 26: The effective throughput of the dynamic $\left(K^{\prime}=2\right)$ and the static $\left(K^{\prime}=0\right)$ cases for $K=4$ and $T=65$ (connections $d$ and $e$ of the asymmetric network of Figure 11).

In our example, the reset technique is applied to the node 7 of the connection $f$. Figures 22, 23, and 24 show that node 7 and connection $f$ restore their performances as in the static case. The remarkable thing is that even the connection $a$ has seen its delay ameliorated due to the load reduction on the node 7 forwarding queue while the throughput of $a$ was not really affected in this example. Hence, after applying the reset technique to node 7 , connections $a$ and $f$ are now classified in the first and the third situations, respectively, where at least 


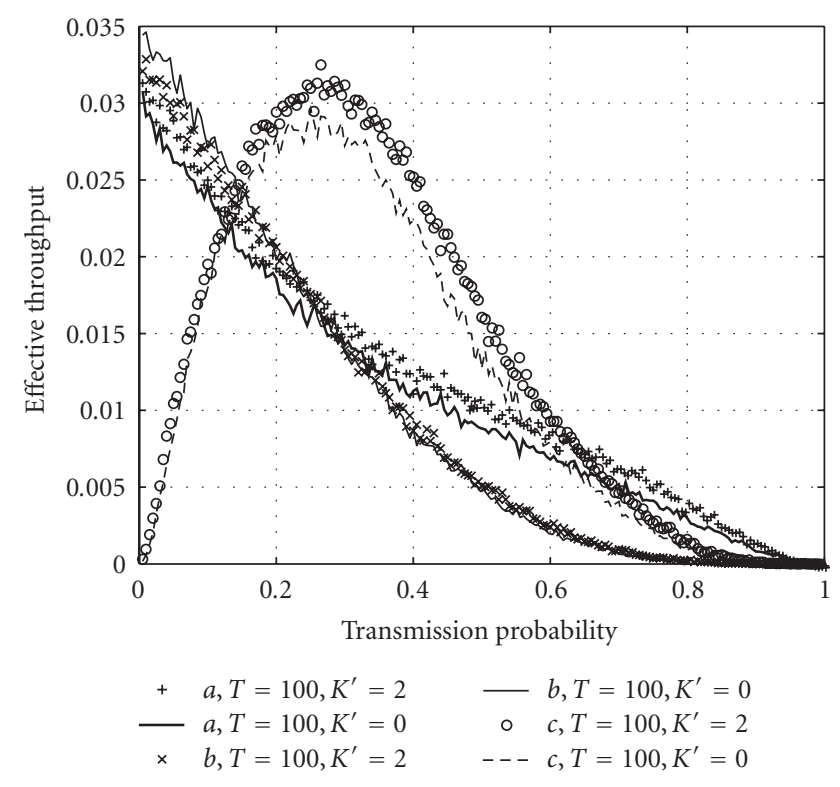

FIgUre 27: The effective throughput of the dynamic $\left(K^{\prime}=2\right)$ and the static $\left(K^{\prime}=0\right)$ cases for $K=4$ and $T=100$ (connections $a, b$, and $c$ of the asymmetric network of Figure 11).

one performance criterion is ameliorated or no deterioration is observed.

\section{PLAYOUT DELAY CONTROL IN VoIP}

After we have seen in general the performances of the scheme, here we study the performances for multimedia applications with delay constraint. Delay, jitter, and packet loss are the main factors impacting audio quality in interactive multimedia applications. In ad hoc network, the audio packets transmitted from a source to a destination can encounter variable delay while crossing the intermediate nodes. In order to play the receiver stream, an application must buffer the packets and play them out after a certain deadline to get again a periodic stream at the application level. Packets arriving after their corresponding deadline are considered lost and are not played out. For that, we need to fix a delay limit needed for some type of application.

Let $T_{s, d}$ be the maximum delay limit for a connection between $s$ and $d$ allowed for each packet in this connection. The instantaneous delay of each packet must not exceed $T_{s, d}$, if so, the destination node must drop this packet. For large $T_{s, d}$, we get less packet dropped and the end-to-end delay may be greater. Then clearly we see the tradeoff generated by setting the value of $T_{s, d}$. The end-to-end effective throughput thpe $e_{s, d}$ of a given connection $R_{s, d}$ can be written as

$$
\text { thpe } e_{s, d}=\operatorname{thp}_{s, d} \cdot P\left(\text { delay } \leq T_{s, d}\right),
$$

where $\operatorname{thp}_{s, d}$ is the end-to-end throughput without the constraint on the delay, and $P\left(\right.$ delay $\left.>T_{s, d}\right)$ is the probability to drop a packet when its delay exceeds $T_{s, d}$.

From Figure 25 to 28 , we simulate two scenarios where in the first one we consider that the delay limit for all

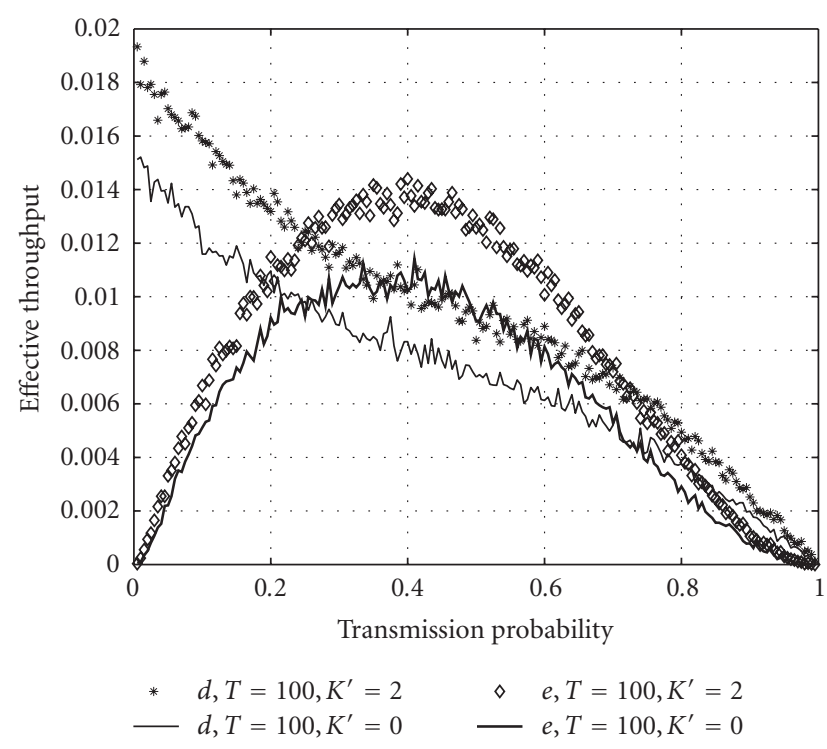

FIGURE 28: The effective throughput of the dynamic $\left(K^{\prime}=2\right)$ and the static $\left(K^{\prime}=0\right)$ cases for $K=4$ and $T=100$ (connections $d$ and $e$ of the asymmetric network of Figure 11).

connections is $T=65$ and in the second one $T=$ 100. The effective throughput shown form these figures is considerably ameliorated in the dynamic scheme, specially for $T=65$, for all connections. While for high-delay limit $T=100$, the performance differences are less for connections $a$ and $c$. It is now clear that it is better to drop packets in intermediate nodes instead of dropping them in the destination due to delay constraint. This is why we have an addition amelioration from Figures 25 and 26. An extension of our scheme can take into consideration the priority of real-time packets. In fact, a high-priority packet can get a minimum limit number of retransmission, not less than the default $K$. Furthermore, it is also interesting to see that $K^{\prime}$ can also be adjusted according to the delay constraint $T_{s, d}$ of a given connection.

\section{CONCLUSION}

In this paper, we have presented a new cross-layer scheme using the limit number of retransmission parameter in a saturated adhoc network, so a dynamic routing can be achieved. The advantage of this scheme consists of the following.

(1) It is a simple distributed cross-layer scheme that can be implemented easily on each node.

(2) It does not need external information about neighboring or other things, but the decision of setting the limit number of retransmission can be taken locally at each node with the help of routing information from the network layer.

(3) The limit number of retransmission has a direct impact on the end-to-end throughput and delay in the saturated network case. 
(4) It mainly controls packets flow by allocating priority to some of them using route information. It is thus efficient in congestion situations.

(5) An advanced configuration of the scheme is possible by also adjusting the step parameter $K^{\prime}$.

The performance evaluation study using analytical and simulation tools has shown that in the case of symmetric linear networks the scheme significantly improves the stability and the throughput for all transmission probabilities. We have also studied the impact of several parameters such as the maximum length of connections and seen that we take benefit from large connections. On the other hand, asymmetric networks performances are directly related to the topology and the neighboring distribution. However, we have identified two properties that a connection must have to get both the throughput and the delay ameliorated. If one of these is not presented, then connection performance can be classified on one of 3 situations where we can benefit or leave unchanged the performances. A reset technique was integrated to the scheme so to optimize performances. In addition, for multimedia applications with delay constraint, we can achieve higher effective throughput as observed in the simulation results.

Moreover, it will be interesting to apply our results in this paper to study its exact behavior using the IEEE 802.11 DCF operation. In fact, an extension of our model presented in this paper is sufficient to study analytically the problem, and some simulations can track the real functioning of the network. It is always possible to enhance the scheme according to the applications need or according to the complexity that we allow. It can be also integrated with other schemes. A content-based dynamic retransmission can be one of the directions to enhance the scheme for video streaming over wireless. In fact, based on the priority of multimedia packet, we can adjust the retransmission limit locally in each node. Therefore, we get a cross-layer-based APP/NETWORK/MAC layers. Improving the QoS of multimedia traffic in multihop ad hoc networks is a real issue, specially, if we need to work on with analytical multilayer model. This is an open issue since high interactions and correlations may exist between different layers in the same node and different nodes in the same network.

\section{REFERENCES}

[1] M. Conti, G. Maselli, G. Turi, and S. Giordano, "Crosslayering in mobile ad hoc network design," Computer, vol. 37, no. 2, pp. 48-51, 2004.

[2] L. Gavrilovska, "Cross-layering approaches in wireless ad hoc networks," Wireless Personal Communications, vol. 37, no. 3-4, pp. 271-290, 2006.

[3] L. Romdhani, Cross layer design of IEEE 802.11-based mobile ad hoc networks for QoS provisioning, Ph.D. thesis, Eurécom Institute, Sophia Antipolis, France, September 2006.

[4] A. A. Kherani, R. El Azouzi, and E. Altman, "Stabilitythroughput tradeoff and routing in multi-hop wireless adhoc networks," in Proceedings of the 5th International IFIP-TC6 Networking Conference, pp. 25-40, Coimbra, Portugal, May 2006.
[5] R. El-Khoury and R. El-Azouzi, "Modeling the effect of forwarding in a multi-hop ad hoc networks with weighted fair queueing," in Proceedings of the 3rd International Conference on Mobile Ad-hoc and Sensor Networks (MSN '07), pp. 5-18, Beijing, China, December 2007.

[6] M. Grossglauser and D. N. C. Tse, "Mobility increases the capacity of ad hoc wireless networks," IEEE/ACM Transactions on Networking, vol. 10, no. 4, pp. 477-486, 2002.

[7] P. Gupta and P. R. Kumar, "The capacity of wireless networks," IEEE Transactions on Information Theory, vol. 46, no. 2, pp. 388-404, 2000.

[8] S. R. Kulkarni and P. Viswanath, "A deterministic approach to throughput scaling in wireless networks," IEEE Transactions on Information Theory, vol. 50, no. 6, pp. 1041-1049, 2004.

[9] L. Tassiulas and A. Ephremides, "Stability properties of constrained queueing systems and scheduling policies for maximum throughput in multihop radio networks," IEEE Transactions on Automatic Control, vol. 37, no. 12, pp. 19361948, 1992.

[10] L. Tassiulas, "Linear complexity algorithms for maximum throughput in radio networks and input queued switches," in Proceedings of the 17th Annual Joint Conference of the IEEE Computer and Communications Societies (INFOCOM '98), vol. 2, pp. 533-539, San Francisco, Calif, USA, March-April 1998.

[11] V. Anantharam, "The stability region of the finite-user slotted ALOHA protocol," IEEE Transactions on Information Theory, vol. 37, no. 3, part 1, pp. 535-540, 1991.

[12] W. Szpankowski, "Stability conditions for some distributed systems: buffered random access systems," Advances in Applied Probability, vol. 26, no. 2, pp. 498-515, 1994.

[13] B. Radunovic and J.-Y. Le Boudec, "Joint scheduling, power control and routing in symmetric, one-dimensional, multihop wireless networks," in Proceedings of the Workshop on Modeling and Optimization in Mobile, Ad Hoc and Wireless Networks (WiOpt'03), Sophia Antipolis, France, March 2003.

[14] X. L. Huang and B. Bensaou, "On max-min fairness and scheduling in wireless ad-hoc networks: analytical framework and implementation," in Proceedings of the 2nd ACM International Symposium on Mobile Ad Hoc Networking \& Computing (MobiHoc'01), pp. 221-231, Long Beach, Calif, USA, October 2001.

[15] L. Tassiulas and S. Sarkar, "Maxmin fair scheduling in wireless networks," in Proceedings of the 21st Annual Joint Conference of the IEEE Computer and Communications Societies (INFOCOM '02), vol. 2, pp. 763-772, New York, NY, USA, June 2002.

[16] N. Bansal and Z. Liu, "Capacity, delay and mobility in wireless ad-hoc networks," in Proceedings of the 22nd Annual Joint Conference of the IEEE Computer and Communications Societies (INFOCOM '03), vol. 2, pp. 1553-1563, San Francisco, Calif, USA, March-April 2003.

[17] S. Toumpis and A. J. Goldsmith, "Large wireless networks under fading, mobility, and delay constraints," in Proceedings of the 23rd Annual Joint Conference of the IEEE Computer and Communications Societies (INFOCOM '04), vol. 1, pp. 609619, Hong Kong, March 2004.

[18] A. El Gamal, J. Mammen, B. Prabhakar, and D. Shah, "Optimal throughput-delay scaling in wireless networks-part I: the fluid model," IEEE/ACM Transactions on Networking, vol. 52, no. 6, pp. 2568-2592, 2006.

[19] M. J. Neely, "Order optimal delay for opportunistic schduling in multi-user wireless uplinks and downlinks," in Proceedings of the 44th Allerton Conference on Communication, Control and Computing, Monticello, Ill, USA, September 2006. 
[20] Z. Jiang and L. Kleinrock, "A packet selection algorithm for adaptive transmission of smoothed video over a wireless channel," Journal of Parallel and Distributed Computing, vol. 60, no. 4, pp. 494-509, 2000.

[21] Q. Li and M. van der Schaar, "Providing adaptive QoS to layered video over wireless local area networks through realtime retry limit adaptation," IEEE Transactions on Multimedia, vol. 6, no. 2, pp. 278-290, 2004.

[22] M.-H. Lu, P. Steenkiste, and T. Chen, "A time-based adaptive retry strategy for video streaming in 802.11 WLANs," Wireless Communications and Mobile Computing, vol. 7, no. 2, pp. 187203, 2007.

[23] S. Mao, S. Lin, Y. Wang, S. Panwar, and Y. Li, "Multipath video transport over wireless ad hoc networks," IEEE Wireless Communications Magazine, vol. 12, no. 4, pp. 42-49, 2005.

[24] Q. Qu, Y. Pei, J. W. Modestino, X. Tian, and B. Wang, "Crosslayer QoS control for video communications over wireless ad hoc networks," EURASIP Journal on Wireless Communications and Networking, vol. 2005, no. 5, pp. 743-756, 2005.

[25] E. Setton, T. Yoo, X. Zhu, A. Goldsmith, and B. Girod, "Crosslayer design of ad hoc networks for real-time video streaming," IEEE Wireless Communications, vol. 12, no. 4, pp. 59-65, 2005.

[26] H. Gharavi and K. Ban, "Dynamic adjustment packet control for video communications over ad-hoc networks," in Proceedings of the IEEE International Conference on Communications (ICC '04), vol. 5, pp. 3086-3090, Paris, France, June 2004.

[27] Y. Yang, J. C. Hou, and L.-C. Kung, "Modeling the effect of transmit power and physical carrier sense in multihop wireless networks," in Proceedings of the 26th Annual Joint Conference of the IEEE Computer and Communications Societies (INFOCOM '07), pp. 2331-2335, Anchorage, Alaska, USA, May 2007. 

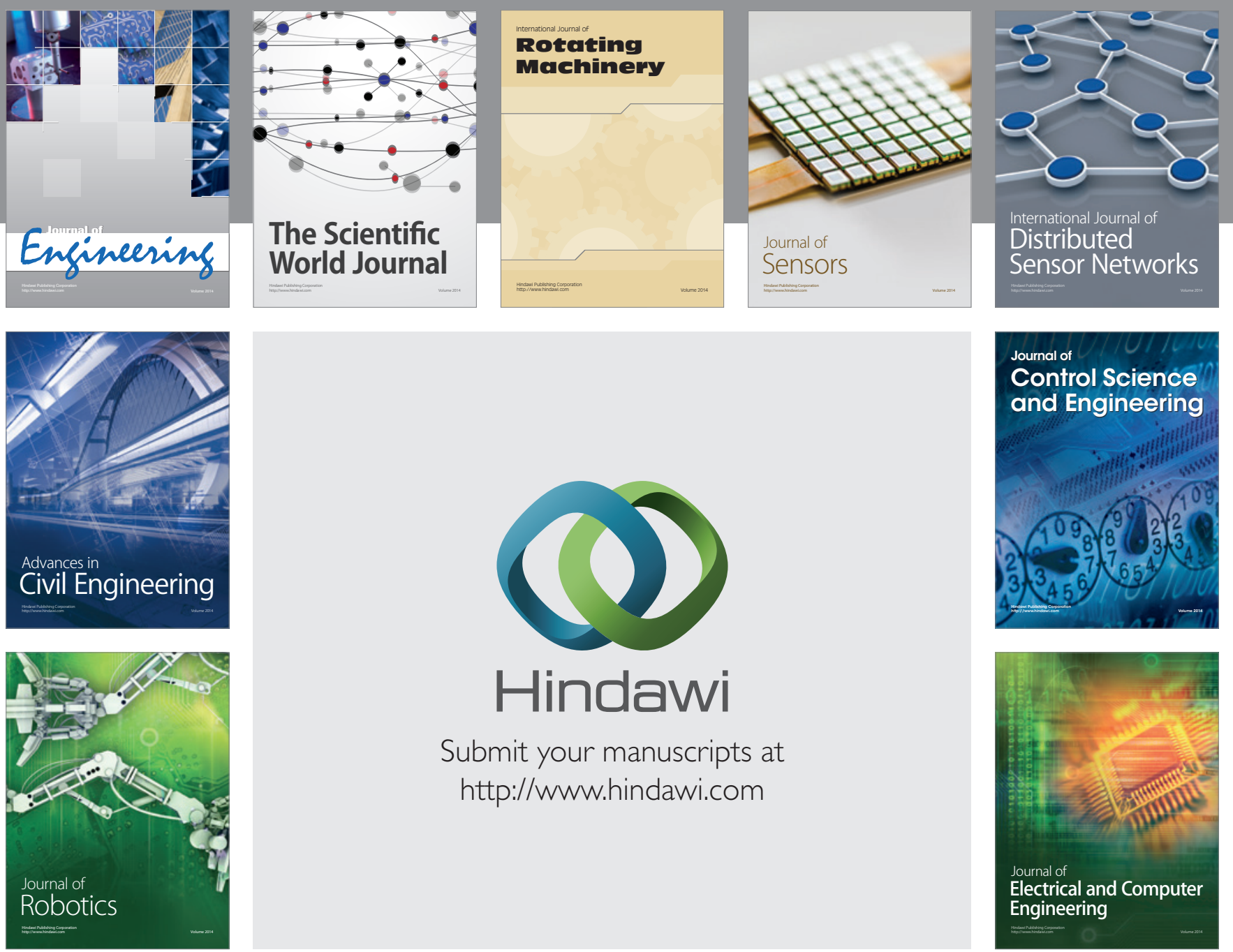

Submit your manuscripts at

http://www.hindawi.com
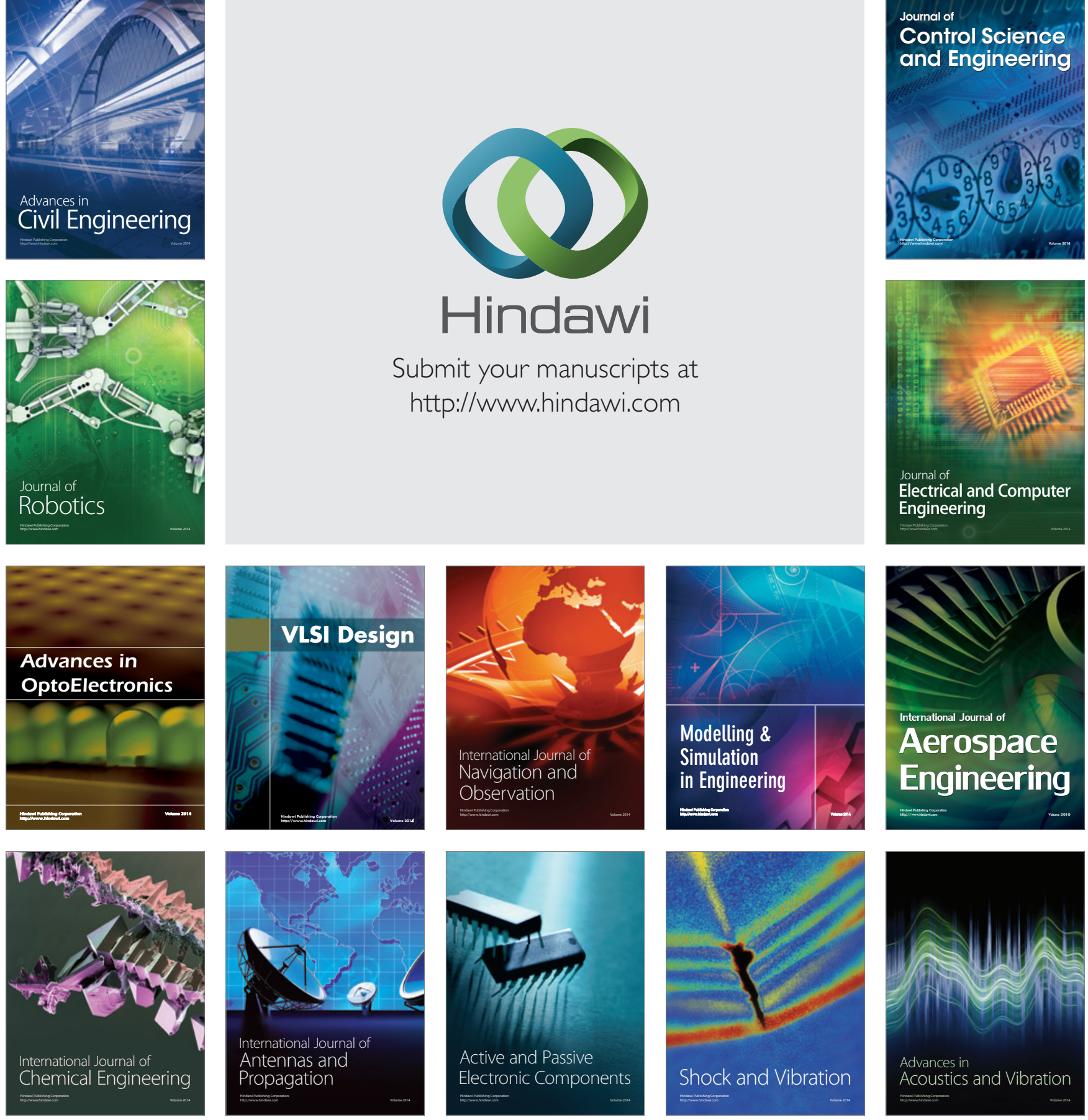